\title{
Numerical solution of time-dependent three-particle Faddeev equations: Calculation of rearrangement $S$ matrices
}

\author{
Zeki C. Kuruoğlu $\oplus^{*}$ \\ Department of Chemistry, Bilkent University, 06800 Bilkent, Ankara, Turkey
}

(Received 23 October 2019; accepted 16 January 2020; published 5 February 2020)

\begin{abstract}
The time-dependent Faddeev equations (TDFEs) are employed for the first time as a computational tool for three-particle scattering problems. Rearrangement transition amplitudes over a wide range of collision energies are extracted from a single numerical wave-packet solution of the TDFE. To numerically solve the TDFE in momentum space for a given initial wave packet, finite-element-type discretizations of Jacobi momenta in terms of local basis functions is employed to convert the TDFE into a set of first-order differential equations in time. Central difference formula for the time derivative is used for the time propagation step. Two forms of TDFE are considered and incorporation of permutational symmetry for three identical particles into these equations is carried out. The proposed method is tested on a three-body model that is often used as a benchmark to compare different computational approaches to three-particle problem. Rearrangement $S$-matrix elements obtained from the analysis of the wave-packet solution of TDFE at asymptotic times are compared with the results of well-established time-independent methods. These results establish that the TDFE approach is a viable and competitive addition to the existing arsenal of computational methods for the three-body scattering problem.
\end{abstract}

DOI: 10.1103/PhysRevC.101.024002

\section{INTRODUCTION}

Computational studies of scattering problems have traditionally been carried out within the time-independent (TI) framework. Differential form of Faddeev equations in coordinate space or Faddeev-AGS integral equations in momentum space has been the usual tool to study the three-body problems in the context of nuclear physics [1-3]. During the last two decades, however, the time-dependent wave-packet (TDWP) approach has emerged as a viable alternative to time-independent approach, especially for reactive scattering of few-atom systems in chemical physics [4-7]. For few-body problems in the context of nuclear physics, although some earlier explorations [8-13] of the time-dependent approach have been made, it has not so far attracted sufficient interest. In this paper, a time-dependent wave-packet method based on the time-dependent Faddeev equations (TDFE) is investigated as a means of solving the scattering problem in a three-particle system with rearrangement and breakup channels. As far as the present author is aware, this is the first time that TDFE is numerically solved.

The time-independent (stationary) approach involves either the solution of the Schrödinger equation in coordinate space subject to scattering boundary conditions, or alternatively, the solution of momentum-space integral equations with kernels possessing singularities corresponding to open channels. Both schemes run into nontrivial complications for scattering systems involving rearrangements and breakup [1,2]. Implementation of appropriate boundary conditions in coordinate space

*kuruoglu@bilkent.edu.tr gives rise to computational complications because (i) there is no single set of coordinates that is capable of expressing boundary conditions in all types of asymptotic regions, (ii) grid discretization or basis expansion using a single set of coordinates is ill suited to describe rearrangement dynamics, and (iii) asymptotic conditions for breakup channel may require prohibitively large computational domains [14,15]. On the other hand, in momentum space approach, kernel singularities associated with breakup channel can be rather difficult to handle computationally.

Some of the problems encountered in time-independent formulation, namely, the non-normalizable nature of scattering wave functions in coordinate space, and complicated singularities of the kernel of integral equations in momentum space may be avoided in the time-dependent (TD) approach, where scattering problem is posed as a time-evolution problem entirely within the Hilbert space. The time-dependent approach is usually implemented in the form of numerical time evolution of a given initial wave packet in coordinate space or momentum space. TD approaches to reactive scattering in the context of chemical physics are usually formulated in coordinate space [4-7]. For few-atom problems within chemical physics, the TDWP approach, compared to the time independent methods, scales more favorably with respect to number of grid points and/or basis functions used to discretize the spatial degrees of freedom. As a result, the TDWP approach to scattering has in fact become a real competitor to time-independent methods for such systems [4,5]. The possibility of using momentum space in time-dependent scattering calculations was explored in Refs. [10-13] in the context of two- and three-particle problems. 
In the TD approach, the time-dependent Schrödinger equation (TDSE) is converted into a set of first-order differential equations in time by discretization of the spatial degrees of freedom. This spatial discretization is achieved by requiring that the TDSE is satisfied, in a weighted-residual sense, either on a set of grid points, or on an approximation space. Time propagation may then be done by a number of well-known algorithms [4-7]. The specification of the approximation space, or of the discretization grid, entails, first, the selection of an appropriate set of coordinates (or momenta). In principle, the approximation space can be built from basis functions in any given set of coordinates. However, the separability of the dynamics in arrangement channels at asymptotic times can not be exploited effectively with a single choice of coordinates.

As is well known from the TI theory, there is no unique set of coordinates capable of describing the four types of asymptotic separable dynamics of a three-particle system. Natural variables to describe asymptotic dynamics are the Jacobi variables for rearrangement channels and hyperspherical variables for the breakup channel. The lack of a single set of coordinates (or momenta) capable of describing all types of asymptotic separable dynamics makes the space discretization a nontrivial problem. Of course, this is the perennial problem of rearrangement scattering whether in TI or TD contexts.

Applications of the TDWP approach in few-atom systems employ either the hyperspherical coordinates [6] or the Jacobi coordinates of a single rearrangement (usually the reactants) $[4,5]$. The use of hyperspherical variables/bases to discretize the spatial degrees of freedom does lead to the challenging problem of transforming between hyperspherical and Jacobi representations of initial and final wave packets. On the other hand, the Jacobi-coordinate approach that uses a grid or basis discretization in reactant Jacobi variables will not be able to describe asymptotic configurations in other arrangements, unless a very fine grid (or an excessively large basis) is employed. Also, when the wave packet is propagated in terms of the Jacobi variables of, say, the initial rearrangement, the task of transforming to the Jacobi variables of other rearrangements can be taxing. In some applications, to account for the presence of reaction and dissociation channels, strategically placed carefully crafted absorbing potentials are used to eliminate the parts of the outgoing wave packet that heads for exit in different rearrangements than the initial.

Another way to approach the coordinate problem would be the simultaneous use of discretization grids or expansion bases in natural variables of all rearrangements. In the context of three-particle problems, Faddeev equations provide a natural setting for this type of approach, in that each Faddeev component can be discretized in terms of its natural Jacobi variables.

In the present work, the time-dependent version of the Faddeev equations (TDFEs) is explored as a tool to implement the time-dependent wave-packet approach for three-particle scattering. Interestingly enough, the time-dependent version of the Faddeev equations appear to have received scant attention in the literature. It appears that the first mention of TDFE is by Kouri et al. in Ref. [16]. Soon thereafter, Evans [17] and Kouri et al. [18] have passingly referred to TDFE while discussing the formal aspects of multiparticle scattering within (what they refer to as) the arrangementchannel quantum mechanics. In another vein, Sultanov and coworkers [19] used TDFE as the starting point to develop a semiclassical approach to Coulombic three-body systems with two heavy and one light particle. The present study appears to be the first TDFE calculation of a three-particle problem above the breakup threshold. In this calculation, the spatial discretization of TDFE is carried out in momentum space, while time is discretized by the central difference formula.

Computational implementations of the time-dependent scattering theory have traditionally been based on the Schrödinger-picture coordinate-space propagation of wave packets. Wave packets move and spread in coordinate space. This gives rise to the so-called boundary-reflection problem, since one has to work in practice with finite approximation spaces, which necessarily have a finite support in coordinate space. Absorbing potentials placed at the edges of the computational domain have been used to combat these complications [4,5]. In contrast, momentum amplitudes of wave packets do not move or spread. A compact momentum-space wave packet remains compact. In other words, the effective momentum-space support (defined, e.g., as the momentum interval over which the probability density is greater than a certain minimum) does not change. Scattering manifests itself in the form of phase modulation of the momentum amplitude of the wave packet. However, wave-packet propagation in momentum representation is not necessarily free of the boundary-reflection problem. The fineness of the momentum discretization determines the size of the corresponding (implicit) region in the coordinate space, and, hence, the maximum period of reflection-free propagation. Nevertheless, the momentum-space approach offers the possibility of extending the duration of the reflection-free propagation by employing a finer momentum grid over only the effective momentum support of the wave packet $[12,13]$.

Organization of this paper is as follows. In Sec. II, the notation and kinematics for three-particle problem is introduced. Section III is devoted to a review of the basic features of the computational time-dependent approach to rearrangement scattering. Time-dependent Faddeev equations are introduced in Sec. IV A. Discretizations of spatial variables and time are the subject of Sec. IV B. Permutational symmetry of three identical particles are incorporated into the TDFE in Sec. V. Computational details and results are reported in Sec. VI. Finally, concluding remarks and possible directions of further research are given in Sec. VII.

\section{THREE-PARTICLE SYSTEM}

\section{A. Kinematics and arrangement channels}

The collision system of interest consists of three particles, which are labeled as 1,2 , and 3. The greek letters $\alpha, \beta$, $\gamma$, etc. will be used as dummy indices for the particles. Adopting the so-called odd-man-out notation, two-fragment partitions $(\alpha)(\beta \gamma)$ of the three particles are referred to as rearrangements and will be enumerated by the index $\alpha$ of the lone (spectator) particle. The quantities associated with a two-particle subsystem $(\beta \gamma)$ will also be labeled by the index 
$\alpha$ of the remaining third particle. The breakup (dissociation) channel (1) (2) (3) will be denoted by the index 0 . The three rearrangement channels and the breakup channel constitute the arrangement channels of the three-particle system.

The Jacobi coordinates for the rearrangement $(\alpha)(\beta \gamma)$ are denoted by $\mathbf{x}_{\alpha}$ and $\mathbf{y}_{\alpha}$. Here, $\mathbf{x}_{\alpha}$ is the internal relative coordinate of the pair $(\beta \gamma)$, and $\mathbf{y}_{\alpha}$ the relative position of the spectator particle $\alpha$ with respect to the center of mass (c.o.m.) of the pair $(\beta \gamma)$. The momenta conjugate to $\mathbf{x}_{\alpha}$ and $\mathbf{y}_{\alpha}$ are $\mathbf{p}_{\alpha}$ and $\mathbf{q}_{\alpha}$, respectively.

The total c.o.m. Hamiltonian $H$ of the system is given as

$$
H=H_{0}+V,
$$

where $H_{0}$ is the kinetic energy operator, and $V$ the total interaction. In the present paper the total interaction is taken to be pairwise additive, viz., $V=V_{1}+V_{2}+V_{3}$.

In terms of the Jacobi variables of rearrangement channels, the free Hamiltonian $H_{0}$ admits the following decompositions:

$$
H_{0}=k_{\alpha}+K_{\alpha}, \quad \alpha=1,2,3,
$$

where

$$
k_{\alpha}=p_{\alpha}^{2} /\left(2 \mu_{\alpha}\right), \quad \text { and } \quad K_{\alpha}=q_{\alpha}^{2} /\left(2 v_{\alpha}\right) .
$$

Here $\mu_{\alpha}$ is the the reduced mass for relative motion within the two-particle subsystem $(\beta \gamma)$ and $v_{\alpha}$ the reduced mass for relative motion of the spectator particle $\alpha$ with respect to c.o.m. of the pair $(\beta, \gamma)$.

The eigenstates of $H_{0}$ are the plane-wave states $\left|\mathbf{p}_{\alpha} \mathbf{q}_{\alpha}\right\rangle$. In labeling state vectors, the notation $\left|\mathbf{p}^{\prime} \mathbf{q}^{\prime}\right\rangle_{\alpha}$ will be used to represent the state $\left|\mathbf{p}_{\alpha}=\mathbf{p}^{\prime}, \mathbf{q}_{\alpha}=\mathbf{q}^{\prime}\right\rangle$. That is, the variables or labels that appear inside a ket vector (or bra vector) are the variables or labels of the rearrangement channel whose index appears as a subscript to the ket (or bra) sign. This notation is especially convenient in the implementation of permutational symmetry when particles are identical. For instance, with $P_{123}$ denoting the even permutation operator that takes (123) to (231), one has $P_{123}|\mathbf{p q}\rangle_{1}=|\mathbf{p q}\rangle_{2}$.

The momentum states are normalized as ${ }_{\alpha}\left\langle\mathbf{p q} \mid \mathbf{p}^{\prime} \mathbf{q}^{\prime}\right\rangle_{\alpha}=$ $\delta\left(\mathbf{p}-\mathbf{p}^{\prime}\right) \delta\left(\mathbf{q}-\mathbf{q}^{\prime}\right)$. Adopting a similar normalization for the position states $|\mathbf{x y}\rangle_{\alpha}$, one has ${ }_{\alpha}\langle\mathbf{x y} \mid \mathbf{p q}\rangle_{\alpha}=(2 / \pi)^{3} \exp \{i \mathbf{p}$. $\mathbf{x}\} \exp \{i \mathbf{q} \cdot \mathbf{y}\}$.

The internal Hamiltonian for the pair $(\beta \gamma)$ is $h_{\alpha}=k_{\alpha}+$ $V_{\alpha}$, where $V_{\alpha}$ is the potential between particles $\beta$ and $\gamma$. The bound states of $h_{\alpha}$ are denoted $\left|\phi_{\alpha n}\right\rangle$ with energies $\epsilon_{\alpha n}$. The time dependence of the bound states is simple: $\left|\phi_{\alpha n}(t)\right\rangle=$ $\exp \left(-i \epsilon_{\alpha n} t\right)\left|\phi_{\alpha n}\right\rangle$.

The asymptotic dynamics in the rearrangement channel $\alpha$ is described by $H_{\alpha}\left(\equiv K_{\alpha}+h_{\alpha}\right)$. The eigenstates of $H_{\alpha}$ (referred to as channel states) will be denoted as $\left|\phi_{\alpha n} q_{\alpha}\right\rangle$ or $\left|\phi_{n} \mathbf{q}\right\rangle_{\alpha}$, with corresponding energy eigenvalues $E_{\alpha n q}=$ $\epsilon_{\alpha n}+q_{\alpha}^{2} / 2 v_{\alpha}$. The channel interaction $V^{\alpha}$ is defined as $V^{\alpha}=$ $H-H_{\alpha}$. As the total interaction is pairwise additive, one has $V^{\alpha}=\sum_{\beta} \bar{\delta}_{\alpha \beta} V_{\beta}$, where $\bar{\delta}_{\alpha \beta}=1-\delta_{\alpha \beta}$.

\section{B. Model}

The three-particle system considered in this work consists of three identical particles (nucleons) whose total interaction is pairwise additive, with the pair potentials restricted to act only on the $s$-wave states of the pairs. The time-dependent approach has been tested using two models for the pair potential:

(i) Rank-1 separable potential

$$
V=|\chi>\lambda<\chi|
$$

where $\langle\mathbf{p} \mid \chi\rangle=1 /\left(p^{2}+c^{2}\right)$, with $c=1.444 \mathrm{fm}^{-1}$. The parameter $\lambda$ was chosen to give the bound-state energy $\epsilon=$ $-0.05370 \mathrm{fm}^{-2}$.

(ii) Malfliet-Tjon (MT-III) potential [20]

$$
V(r)=V_{R} \frac{e^{-\mu_{R} r}}{r}-V_{A} \frac{e^{-\mu_{A} r}}{r},
$$

whose momentum-space representation is given as

$$
V\left(\mathbf{q}, \mathbf{q}^{\prime}\right)=\frac{1}{2 \pi^{2}}\left(\frac{V_{R}}{\left(\mathbf{q}-\mathbf{q}^{\prime}\right)^{2}+\mu_{R}^{2}}-\frac{V_{A}}{\left(\mathbf{q}-\mathbf{q}^{\prime}\right)^{2}+\mu_{A}^{2}}\right) .
$$

The values of parameters are taken from [21]: $V_{A}=626.885$ $\mathrm{MeV} \mathrm{fm}, V_{R}=1438.72 \mathrm{MeV} \mathrm{fm}, \mu_{A}=1.55 \mathrm{fm}^{-1}$, and $\mu_{R}=$ $3.11 \mathrm{fm}^{-1}$. The particle (nucleon) mass and $\hbar$ are set to unity and $\mathrm{fm}$ is taken as as the unit of length. The nucleon mass adopted yields the conversion factor $1 \mathrm{fm}^{-2}=41.47 \mathrm{MeV}$.

In the present paper, attention is further restricted to states of zero total angular momentum. Therefore, in what follows, all angular variables will disappear and momentum states $|\mathbf{p q}\rangle_{\alpha}$ will be replaced by spherical momentum states $|p q\rangle_{\alpha}\left(\equiv(4 \pi)^{-1} \int d \hat{\mathbf{p}} \int d \hat{\mathbf{q}}|\mathbf{p q}\rangle_{\alpha}\right)$. The subspace of all possible asymptotic states $\left|\phi_{n} q\right\rangle_{\alpha}$ in a given rearrangement $\alpha$ of the three-particle model is then characterized by the projector

$$
\Pi_{\alpha}=\sum_{n} \int q^{2} d q\left|\phi_{n} q\right\rangle_{\alpha}{ }_{\alpha}\left\langle\phi_{n} q\right|
$$

As is well known, with separable pair potentials, Faddeev equations for rearrangement transition operators are reduced to a set of effective two-body integral equations in the spectator momenta. Although these effective potentials possess logarithmic singularities that require careful treatment, various numerical techniques exist to solve them accurately. In the present work, reference results for the separable-potential model were obtained by solving the effective two-body equations using a Schwinger-type variational method.

For the other model with MT-III potential, accurate benchmark solutions above the breakup threshold have been reported in the literature using several different approaches [21-23]. However, these benchmark results are available only at two collision energies. Reference results over a wide range of energies were generated using an extension of the coupledreaction-channels (CRC) method in which the CRC ansatz is augmented by two-body pseudostates to simulate the breakup channel. It has been demonstrated in the past that this method can give accurate results for both the separable-potential model [24] and the local MT-III model [25].

\section{Approximation spaces}

Discretization of spatial variables is usually carried out by projecting the wave packet on an approximation space and then requiring the TDSE (or equivalent dynamical equation) to hold on a test space. Depending on the nature of the 
selected approximation and test spaces, one can have different computational schemes. In this work, the Galerkin scheme was chosen to handle the spatial degrees of freedom.

The basis functions for the $\alpha$-rearrangement subspace $\mathcal{S}_{\alpha}$ are the direct product functions $u_{\alpha i}\left(p_{\alpha}\right) v_{\alpha j}\left(q_{\alpha}\right), i=$ $1,2, \ldots, I_{\alpha} ; j=1,2, \ldots, J_{\alpha}$. Here $\left\{u_{\alpha i}\left(p_{\alpha}\right)\right\}$ is a suitable set of $I_{\alpha}$ expansion functions for the pair, while $\left\{v_{\alpha j}\left(q_{\alpha}\right)\right\}$ a suitable set of $J_{\alpha}$ expansion functions for the spectator.

Note that basis functions in a given variable are not necessarily orthonormal. Therefore, one needs the overlap matrices $\boldsymbol{\Delta}_{\alpha p}$ and $\boldsymbol{\Delta}_{\alpha q}$ for the bases $\left\{u_{i}\left(p_{\alpha}\right)\right\}$ and $\left\{u_{j}\left(q_{\alpha}\right)\right\}$, respectively. The overlaps ${ }_{\alpha}\left\langle u_{i} v_{j} \mid u_{i^{\prime}} v_{j^{\prime}}\right\rangle_{\alpha}$ are collected in the matrix $\boldsymbol{\Delta}_{\alpha}$. which is given by the direct product of matrices $\boldsymbol{\Delta}_{\alpha p}$ and $\boldsymbol{\Delta}_{\alpha q}$. Due to its direct-product nature, this overlap matrix is easy to deal with.

\section{TIME-DEPENDENT DESCRIPTION OF REARRANGEMENT SCATTERING IN A THREE-PARTICLE SYSTEM}

Time-dependent treatment of the collision of an incident particle with a bound pair seeks the solution of the timedependent Schrödinger equation,

$$
i \frac{\partial|\Psi(t)\rangle}{\partial t}=H|\Psi(t)\rangle
$$

subject to the initial condition

$$
|\Psi(0)\rangle=\left|\Phi_{\alpha n_{0} q_{0}}(0)\right\rangle=\left|\phi_{\alpha n_{0}} f_{\alpha q_{0}}\right\rangle=\left|\phi_{n_{0}} f_{q_{0}}\right\rangle_{\alpha}
$$

describing the spectator particle $\alpha$ (with average momentum $q_{0}$ ) incident upon the pair $(\beta \gamma)$ (in bound state $\phi_{\alpha n_{0}}$ ). Here $\left|f_{\alpha q_{0}}\right\rangle$ is an incoming wave packet for the relative motion of particle $\alpha$ with average momentum $q_{0}$, and average separation $y_{0}$. The present numerical implementation uses a Gaussian wave packet:

$$
\left\langle y_{\alpha} \mid f_{\alpha q_{0}}\right\rangle=N \frac{e^{i q_{0} y_{\alpha}}}{y_{\alpha}} e^{-\left(y_{\alpha}-y_{0}\right)^{2} / 2 d^{2}},
$$

where $N$ is a normalization constant, and $d$ the width parameter. The average separation $y_{0}$ and the width $d$ are chosen so that the effective coordinate-space support of the initial wave packet is outside the the range of the channel interaction $\left(V^{\alpha}\right)$.

The free time evolution of the initial state $\left|\Phi_{\alpha v_{0} q_{0}}\right\rangle$ under $H_{\alpha}$ is given by

$$
\left|\Phi_{\alpha n_{0} q_{0}}(t)\right\rangle=\left|\phi_{n_{0}}(t) f_{q_{0}}(t)\right\rangle_{\alpha}=\int q^{2} d q\left|\phi_{n_{0}} q\right\rangle_{\alpha} f_{\alpha q_{0}}(q, t),
$$

where $f_{\alpha q_{0}}(q, t)=e^{-i t E_{\alpha n_{0} q}} f_{\alpha q_{0}}(q)$, with $E_{\alpha n_{0} q}=\epsilon_{\alpha n_{0}}+$ $q^{2} / 2 v_{\alpha}$, and $f_{\alpha q_{0}}(q)$ being the momentum amplitude of the Gaussian wave packet given in Eq. (7).

Let $\left|\Psi_{\alpha n_{0} q_{0}}(t)\right\rangle$ denote the solution of the three-particle TDSE that coincide with $\left|\Phi_{\alpha v_{0} q_{0}}(t)\right\rangle$ at time $t=0$. One can define the rearrangement components of the total wave packet via the projections

$$
\left|\Psi_{\alpha n_{0} q_{0}}^{(\beta)}(t)\right\rangle=\Pi_{\beta}\left|\Psi_{\alpha n_{0} q_{0}}(t)\right\rangle, \quad \beta=1,2,3 .
$$

As $t \rightarrow \infty$, these components will be spatially separated, and the scattering into the rearrangement $\beta$ will be solely contained in $\left|\Psi_{\alpha n_{0} q_{0}}^{(\beta)}\right\rangle$. The components $\left|\Psi_{\alpha n_{0} q_{0}}^{(\beta)}(t)\right\rangle, \beta=1,2,3$, can be further analyzed as

$$
\left|\Psi_{\alpha n_{0} q_{0}}^{(\beta)}(t)\right\rangle=\sum_{n} \int q^{2} d q\left|\phi_{n} q\right\rangle_{\beta} g_{\beta n}(q, t),
$$

where

$$
g_{\beta n}(q, t)={ }_{\beta}\left\langle\phi_{n} q \mid \Psi_{\alpha n_{0} q_{0}}^{(\beta)}(t)\right\rangle .
$$

As $t \rightarrow \infty, g_{\beta n}(q, t)$ represents an outgoing wave packet for the spectator particle $\beta$.

The fundamental result of the time-dependent scattering theory is that, as $t \rightarrow \infty$,

$$
\left|\Psi_{\alpha n_{0} q_{0}}^{(\beta)}(t)\right\rangle=S_{\beta \alpha}\left|\Phi_{\alpha n_{0} q_{0}}(t)\right\rangle, \quad \beta=1,2,3, \quad \alpha=1,2,3,
$$

where $S_{\beta \alpha}$ is the scattering operator for the rearrangement process $(\alpha)(\beta \gamma) \rightarrow(\beta)(\alpha \gamma)$.

To extract the sharp-energy matrix elements of the rearrangement $S$ operators, one can use the energy conserving property of $S_{\beta \alpha}$, viz.,

$$
{ }_{\beta}\left\langle\phi_{n^{\prime} q^{\prime}}\left|S_{\beta \alpha}\right| \phi_{n q}\right\rangle_{\alpha}=\hat{S}_{\beta n^{\prime} q^{\prime}, \alpha n q}\left(E_{\alpha n q}\right) \delta\left(E_{\beta n^{\prime} q^{\prime}}-E_{\alpha n q}\right),
$$

where $\hat{S}_{\beta n^{\prime} q^{\prime}, \alpha n q}\left(E_{\alpha n q}\right)$ is the reduced $S$-matrix element whose absolute value square gives the probability for the transition from the initial state $\left|\phi_{n} q\right\rangle_{\alpha}$ to the final state $\left|\phi_{n^{\prime}} q^{\prime}\right\rangle_{\beta}$. By projecting Eq. (10) onto the channel state $\left|\phi_{n^{\prime}} q^{\prime}\right\rangle_{\beta}$, and making use of Eqs. (8) and (12), one finds, as $t \rightarrow \infty$,

$$
\begin{aligned}
\hat{S}_{\beta n^{\prime} q^{\prime}, \alpha n_{0} q}(E) & =\sqrt{\frac{v_{\beta} q^{\prime}}{v_{\alpha} q}} \frac{{ }_{\beta}\left\langle\phi_{n^{\prime}} q^{\prime} \mid \Psi_{\alpha n_{0} q_{0}}(t)\right\rangle}{{ }_{\alpha}\left\langle\phi_{n_{0}} q \mid \Phi_{\alpha n_{0} q_{0}}(t)\right\rangle} \\
& =\sqrt{\frac{v_{\beta} q^{\prime}}{v_{\alpha} q}} \frac{g_{\beta n^{\prime}}\left(q^{\prime}, t\right)}{f_{\alpha q_{0}}(q, t)},
\end{aligned}
$$

where $q$ is fixed by the requirement $E=\epsilon_{\beta n^{\prime}}+q^{2} / 2 v_{\beta}=$ $\epsilon_{\alpha n_{0}}+q^{2} / 2 v_{\alpha}$.

Numerical determination of $\Psi_{\alpha n_{0} q_{0}}(t)$ is beset by the wellknown difficulties of rearrangement scattering, as alluded to in Sec. I. The usual strategy to tackle the time-dependent propagation of a wave packet would be to discretize the the spatial degrees of freedom by expanding the wave packet in a basis. This basis could be specified either in momentum space or in coordinate space. In this work, momentum space is used. Choosing a suitable basis, however, is no easy task, and is hampered by the well-known complications of the rearrangement scattering. For instance, if three-particle basis functions are taken as direct products of functions in $p_{\alpha}$ and $q_{\alpha}$, such a separable basis will not be appropriate to describe the dynamics in other rearrangement channels $\beta(\neq \alpha)$.

Clearly the chosen basis must be capable of describing outgoing (separable) wave packets such as $\left|\phi_{\beta n} g_{\beta n}\right\rangle$ in all rearrangements, $\beta=1,2,3$. In earlier work $[10,11]$, inspired from the coupled-reaction-channels (CRC) method, a nonorthogonal basis $\left\{u_{\alpha i}\left(q_{\alpha}\right) v_{\alpha j}\left(q_{\alpha}\right), n=1,2, \ldots, I_{\alpha}, m=\right.$ $\left.1,2, \ldots, J_{\alpha}, \alpha=1,2,3\right\}$, consisting of separable basis functions from all rearrangements had been used. In this CRC-like 
scheme, the total wave packet was expanded as

$$
\left|\Psi_{\alpha n_{0} q_{0}}(t)\right\rangle=\sum_{\beta=1}^{3} \sum_{i=1}^{I_{\beta}} \sum_{j=1}^{J_{\beta}}\left|u_{i} v_{j}\right\rangle_{\beta} c_{\beta i j}(t)
$$

and expansion coefficients $c_{\beta i j}(t)$ are obtained by solving the following system of first-order equations:

$$
\begin{aligned}
& i \sum_{\beta=1}^{3} \sum_{i^{\prime}=1}^{I_{\beta}} \sum_{j^{\prime}=1}^{J_{\beta}} \gamma\left\langle u_{i} v_{j} \mid u_{i^{\prime}} v_{j^{\prime}}\right\rangle_{\beta} \frac{d c_{\beta i^{\prime} j^{\prime}}(t)}{d t} \\
& \quad=\sum_{\beta=1}^{3} \sum_{i^{\prime}=1}^{I_{\beta}} \sum_{j^{\prime}=1}^{J_{\beta}} \gamma\left\langle u_{i} v_{j}|H| u_{i^{\prime}} v_{j^{\prime}}\right\rangle_{\beta} c_{\beta i^{\prime} j^{\prime}}(t),
\end{aligned}
$$

where $i=1, \ldots, I_{\gamma}, j=1, \ldots, J_{\gamma}$ and $\gamma=1,2,3$. Although this scheme was shown to work reasonably well with modest basis sizes $[10,11,13]$, the presence of the nonorthogonality overlap matrix ${ }_{\alpha}\left\langle u_{i} v_{j} \mid u_{i^{\prime}} v_{j^{\prime}}\right\rangle_{\beta}$ is an undesirable feature that makes the numerical solution of Eq. (16) awkward and inconvenient.

The use of basis functions in hyperspherical coordinates provides an alternative to separable bases in Jacobi coordinates (see, e.g., Ref. [6]). However, description of separable wave packets such as $\left|\phi_{\beta n} g_{\beta n}\right\rangle$ in terms of hyperspherical coordinates is not free of difficulties either. The description of a wave packet that splits into rearrangement pieces, which emerge in different asymptotic regions is best done in terms of separable bases in Jacobi coordinates. Thus simultaneous use of multiarrangement bases without giving rise to nonorthogonality problem is called for. Faddeev formalism of three particle dynamics provides just the right setting for this approach.

\section{TIME-DEPENDENT FADDEEV EQUATIONS}

\section{A. Formalism}

The celebrated Faddeev equations [26] for the threeparticle system have been originally derived and used within time-independent framework. It involves dividing the the total time-independent scattering state $\left|\Psi_{E}\right\rangle$ for total energy $E$ as $\left|\Psi_{E}\right\rangle=\left|\psi_{1}^{F}\right\rangle+\left|\psi_{2}^{F}\right\rangle+\left|\psi_{3}^{F}\right\rangle$, in terms of the three rearrangement components $\left|\psi_{\alpha}^{F}\right\rangle, \alpha=1,2,3$. These components satisfy the coupled equations

$$
\begin{gathered}
\left(\begin{array}{ccc}
E-H_{1} & 0 & 0 \\
0 & E-H_{2} & 0 \\
0 & 0 & E-H_{3}
\end{array}\right)\left(\begin{array}{l}
\left|\psi_{1}^{F}\right\rangle \\
\left|\psi_{2}^{F}\right\rangle \\
\left|\psi_{3}^{F}\right\rangle
\end{array}\right) \\
=\left(\begin{array}{ccc}
0 & V_{1} & V_{1} \\
V_{2} & 0 & V_{2} \\
V_{3} & V_{3} & 0
\end{array}\right)\left(\begin{array}{l}
\left|\psi_{1}^{F}\right\rangle \\
\left|\psi_{2}^{F}\right\rangle \\
\left|\psi_{3}^{F}\right\rangle
\end{array}\right) .
\end{gathered}
$$

Another lesser-known version of Faddeev wave function equations is

$$
\begin{gathered}
\left(\begin{array}{ccc}
E-H_{1} & 0 & 0 \\
0 & E-H_{2} & 0 \\
0 & 0 & E-H_{3}
\end{array}\right)\left(\begin{array}{l}
\left|\Psi_{1}^{T F}\right\rangle \\
\left|\Psi_{2}^{T F}\right\rangle \\
\left|\Psi_{3}^{T F}\right\rangle
\end{array}\right) \\
=\left(\begin{array}{ccc}
0 & V_{2} & V_{3} \\
V_{1} & 0 & V_{3} \\
V_{1} & V_{2} & 0
\end{array}\right)\left(\begin{array}{l}
\left|\Psi_{1}^{T F}\right\rangle \\
\left|\Psi_{2}^{T F}\right\rangle \\
\left|\Psi_{3}^{T F}\right\rangle
\end{array}\right),
\end{gathered}
$$

where the interaction matrix is the transpose of the usual Faddeev interaction matrix of Eq. (17). This version will be referred to as the transposed Faddeev equations. As elaborated by Levin [27], this version corresponds to the FaddeevLovelace choice for the coupling of rearrangements. Each of the components $\left|\Psi_{1}^{T F}\right\rangle,\left|\Psi_{2}^{T F}\right\rangle$, and $\left|\Psi_{3}^{T F}\right\rangle$ are, in fact, identical to the full state $\left|\Psi_{E}\right\rangle$. To obtain Eq. (18), one first rewrites the full Schrödinger equation as

$$
\left(E-H_{1}\right)\left|\Psi_{E}\right\rangle=V_{2}\left|\Psi_{E}\right\rangle+V_{3}\left|\Psi_{E}\right\rangle,
$$

and then labels different occurences of $\left|\Psi_{E}\right\rangle$ with rearrangement indices in the following manner:

$$
\left(E-H_{1}\right)\left|\Psi_{1}^{T F}\right\rangle=V_{2}\left|\Psi_{2}^{T F}\right\rangle+V_{3}\left|\Psi_{3}^{T F}\right\rangle .
$$

That is, the arrangement subscript $\alpha$ in $\left|\Psi_{\alpha}^{T F}\right\rangle$ is simply a bookkeeping device indicating that, in expressions such as $\left(E-H_{\alpha}\right)\left|\Psi_{E}\right\rangle$ or $V_{\alpha}\left|\Psi_{E}\right\rangle$, the total scattering state $\left|\Psi_{E}\right\rangle$ will be treated in terms of the Jacobi variables and/or expansion basis appropriate for the rearrangement $\alpha$.

In the time-dependent context, the full wave packet $|\Psi(t)\rangle$ is decomposed into Faddeev components as

$$
|\Psi(t)\rangle=\left|\psi_{1}^{F}(t)\right\rangle+\left|\psi_{2}^{F}(t)\right\rangle+\left|\psi_{3}^{F}(t)\right\rangle,
$$

where the time-dependent Faddeev components are now defined via

$$
i \frac{\partial\left|\psi_{\beta}^{F}(t)\right\rangle}{\partial t}=V_{\beta}|\Psi(t)\rangle
$$

with $\beta=1,2,3$. This is in complete analogy to $(E-$ $\left.H_{0}\right)\left|\psi_{\beta}^{F}\right\rangle=V_{\beta}\left|\Psi_{E}\right\rangle$ in the time-independent context. Since one assumes that $V$ is pairwise additive, viz., $V=V_{1}+V_{2}+$ $V_{3}$, one can easily verify that summing Eq. (20) over the components gives the time-dependent Schrödinger equation. If one rewrites Eq. (20) as

$$
\left(i \frac{\partial}{\partial t}-H_{0}\right)\left|\psi_{\beta}^{F}(t)\right\rangle=V_{\beta}\left(\left|\psi_{1}^{F}(t)\right\rangle+\left|\psi_{2}^{F}(t)\right\rangle+\left|\psi_{3}^{F}(t)\right|\right)
$$

and then rearranges, one obtains the TDFE:

$$
i \frac{\partial\left|\psi_{\beta}^{F}(t)\right\rangle}{\partial t}=H_{\beta}\left|\psi_{\beta}^{F}\right\rangle+V_{\beta}\left(\left|\psi_{\gamma}^{F}(t)\right\rangle+\left|\psi_{\alpha}^{F}(t)\right|\right),
$$

where $(\alpha, \beta, \gamma)$ stand for cyclic permutations of (1,2,3). For future reference, Eq. (22) is rewritten in explicit matrix form:

$$
\begin{aligned}
i \frac{\partial}{\partial t}\left(\begin{array}{l}
\left|\psi_{1}^{F}(t)\right\rangle \\
\left|\psi_{2}^{F}(t)\right\rangle \\
\left|\psi_{3}^{F}(t)\right\rangle
\end{array}\right)= & {\left[\left(\begin{array}{ccc}
H_{1} & 0 & 0 \\
0 & H_{2} & 0 \\
0 & 0 & H_{3}
\end{array}\right)\right.} \\
& \left.+\left(\begin{array}{ccc}
0 & V_{1} & V_{1} \\
V_{2} & 0 & V_{2} \\
V_{3} & V_{3} & 0
\end{array}\right)\right]\left(\begin{array}{l}
\left|\psi_{1}^{F}(t)\right\rangle \\
\left|\psi_{2}^{F}(t)\right\rangle \\
\left|\psi_{3}^{F}(t)\right\rangle
\end{array}\right) .
\end{aligned}
$$

TDFE is to be solved subject to the initial condition

$$
\left|\psi_{\beta}^{F}(0)\right\rangle=\delta_{\beta \alpha}\left|\Phi_{\alpha n_{0} q_{0}}(0)\right\rangle,
$$

where $\Phi_{\alpha n_{0} q_{0}}$ is the initial wave packet defined in Eq. (6). Note that, in our notation for Faddeev components, reference to the initial state has been suppressed. 
As shown in Ref. [16], at asymptotic times, the outgoing wave packet emerging in the rearrangement $\beta$ is solely contained in the Faddeev component $\left|\psi_{\beta}^{F}(t)\right\rangle$. That is, as $t \rightarrow \infty$,

$$
\Pi_{\beta}\left|\psi_{\beta}^{F}(t)\right\rangle=S_{\beta \alpha}\left|\Phi_{\alpha n_{0} q_{0}}(t)\right\rangle, \quad \beta=1,2,3, \quad \alpha=1,2,3 .
$$

and

$$
\hat{S}_{\beta n^{\prime} q^{\prime}, \alpha n_{0} q}(E)=\sqrt{\frac{v_{\beta} q^{\prime}}{v_{\alpha} q}} \frac{{ }_{\beta}\left\langle\phi_{n^{\prime}} q^{\prime} \mid \psi_{\beta}^{F}(t)\right\rangle}{{ }_{\alpha}\left\langle\phi_{n_{0}} q \mid \Phi_{\alpha n_{0} q_{0}}(t)\right\rangle} .
$$

Alternatively, one can extract the rearrangement $S$ matrix using Eq. (14) with the total wave packet $\Psi_{\alpha n_{0} q_{0}}(t)$ obtained from the Faddeev components via $\Psi_{\alpha n_{0} q_{0}}(t)=\sum_{\beta=1}^{3} \psi_{\beta}^{F}(t)$. In other words, at asymptotic future, both $\Pi_{\beta} \Psi_{\alpha n_{0} q_{0}}(t)$ and $\Pi_{\beta} \psi_{\beta}^{F}(t)$ represent the portion of the outgoing wave packet in which spectator $\beta$ is flying away from the bound pair $(\alpha \gamma)$.

The time-dependent version of the transposed Faddeev equations read

$$
\begin{aligned}
i \frac{\partial}{\partial t}\left(\begin{array}{l}
\left|\Psi_{1}^{T F}(t)\right\rangle \\
\left|\Psi_{2}^{T F}(t)\right\rangle \\
\left|\Psi_{3}^{T F}(t)\right\rangle
\end{array}\right)= & {\left[\left(\begin{array}{ccc}
H_{1} & 0 & 0 \\
0 & H_{2} & 0 \\
0 & 0 & H_{3}
\end{array}\right)+\left(\begin{array}{ccc}
0 & V_{2} & V_{3} \\
V_{1} & 0 & V_{3} \\
V_{1} & V_{2} & 0
\end{array}\right)\right] } \\
& \times\left(\begin{array}{l}
\left|\Psi_{1}^{T F}(t)\right\rangle \\
\left|\Psi_{T F}^{F}(t)\right\rangle \\
\left|\Psi_{3}^{T F}(t)\right\rangle
\end{array}\right) .
\end{aligned}
$$

where $\left|\Psi_{1}^{T F}(t)\right\rangle=\left|\Psi_{2}^{T F}(t)\right\rangle=\left|\Psi_{3}^{T F}(t)\right\rangle=|\Psi(t)\rangle$. This set of equations will be referred to as time-dependent transposed Faddeev equations (TDTFE). The initial conditions for TDTFE are

$$
\left|\Psi_{1}^{F L}(0)\right\rangle=\left|\Psi_{2}^{F L}(0)\right\rangle=\left|\Psi_{3}^{F L}(0)\right\rangle=\left|\Phi_{\alpha n_{0} q_{0}}(0)\right\rangle,
$$

where $\alpha$ is the initial rearrangement.

In principle any one of $\Psi_{\beta}^{T F}(t), \beta=1,2,3$, can be used as the full wave packet $\Psi(t)$ in Eq. (14). In practice, however, due to the finite nature of grid or basis representations, scattering into the rearrangement $\beta$ will be best represented by $\Psi_{\beta}^{T F}(t)$. Thus, in computational implementation of the TDTFE, asymptotic analysis can be based on

$$
\Pi_{\beta}\left|\Psi_{\beta}^{T F}(t)\right\rangle=S_{\beta \alpha}\left|\Phi_{\alpha n_{0} q_{0}}(t)\right\rangle .
$$

and

$$
\hat{S}_{\beta n^{\prime} q^{\prime}, \alpha n_{0} q}(E)=\sqrt{\frac{v_{\beta} q^{\prime}}{v_{\alpha} q}} \frac{{ }_{\beta}\left\langle\phi_{n^{\prime}} q^{\prime} \mid \Psi_{\beta}^{T F}(t)\right\rangle}{{ }_{\alpha}\left\langle\phi_{n_{0}} q \mid \Phi_{\alpha n_{0} q_{0}}(t)\right\rangle},
$$

for $t$ in asymptotic future.

\section{B. Discretizaton of spatial variables and time in TDFE}

Structure of TDFE is especially suited to the simultaneous use of all three rearrangement bases. Each component $\psi_{\beta}^{F}(t)$ is expanded in its natural separable basis $\left\{\left|u_{i} v_{j}\right\rangle_{\beta}\right\}$, viz.,

$$
\left|\psi_{\beta}^{F}(t)\right\rangle=\sum_{i=1}^{I_{\beta}} \sum_{j=1}^{J_{\beta}}\left|u_{i} v_{j}\right\rangle_{\beta} c_{\beta i j}^{F}(t),
$$

with initial condition in Eq. (24) becoming

$$
c_{\beta i j}^{F}(0)=\delta_{\beta \alpha} \sum_{i^{\prime}=1}^{I_{\alpha}} \sum_{j^{\prime}=1}^{J_{\alpha}}\left(\boldsymbol{\Delta}_{\alpha}^{-1}\right)_{i j, i^{\prime} j^{\prime}} \alpha\left\langle u_{i^{\prime}} v_{j^{\prime}} \mid \Phi_{\alpha n_{0} q_{0}}(0)\right\rangle .
$$

To obtain the time-evolution equation for the expansion coefficients, the weighted-residual approach can be used. To this end, one introduces an error term (or residual) for each rearrangement component via

$$
\begin{aligned}
\left|\varepsilon_{\alpha}\right\rangle= & {\left[i \frac{\partial}{\partial t}-H_{\alpha}\right] \sum_{i=1}^{I_{\alpha}} \sum_{j=1}^{J_{\alpha}}\left|u_{i} v_{j}\right\rangle_{\alpha} c_{\alpha i j}^{F}(t) } \\
& -V_{\alpha} \sum_{i=1}^{I_{\beta}} \sum_{j=1}^{J_{\beta}}\left|u_{i} v_{j}\right\rangle_{\beta} c_{\beta i j}^{F}(t)-V_{\alpha} \sum_{i=1}^{I_{\gamma}} \sum_{j=1}^{J_{\gamma}}\left|u_{i} v_{j}\right\rangle_{\gamma} c_{\gamma i j}^{F}(t) .
\end{aligned}
$$

Galerkin method requires that the error term $\left|\varepsilon_{\alpha}\right\rangle$ be orthogonal to the approximation subspace $\mathcal{S}_{\alpha}$, viz., ${ }_{\alpha}\left\langle u_{i} v_{j} \mid \varepsilon_{\alpha}\right\rangle=$ 0 . On the other hand, collocation method would require ${ }_{\alpha}\left\langle p_{i} q_{j} \mid \varepsilon_{\alpha}\right\rangle=0$. The time-evolution equations that follow from the Galerkin scheme read

$$
\boldsymbol{\Delta}_{\alpha} \frac{d \mathbf{C}_{\alpha}^{F}(t)}{d t}=\mathbf{H}_{\alpha} \mathbf{C}_{\alpha}^{F}(t)+\sum_{\beta} \bar{\delta}_{\alpha \beta} \mathbf{V}_{\alpha \beta}^{F} \mathbf{C}_{\beta}^{F}(t),
$$

where $\mathbf{C}_{\alpha}^{F}(t)$ is the column vector of expansion coefficients $c_{\alpha i j}^{F}(t)$, and

$$
\begin{aligned}
\left(\mathbf{H}_{\alpha}\right)_{i j, i^{\prime} j^{\prime}} & ={ }_{\alpha}\left\langle u_{i} v_{j}\left|H_{\alpha}\right| u_{i^{\prime}} v_{j^{\prime}}\right\rangle_{\alpha} \\
\left(\mathbf{V}_{\alpha \beta}^{F}\right)_{i j, i^{\prime} j^{\prime}} & ={ }_{\alpha}\left\langle u_{i} v_{j}\left|V_{\alpha}\right| u_{i^{\prime}} v_{j^{\prime}}\right\rangle_{\beta} .
\end{aligned}
$$

By defining

$$
\begin{aligned}
\mathbf{C}^{F}(t) & =\left(\begin{array}{l}
\mathbf{C}_{1}^{F}(t) \\
\mathbf{C}_{2}^{F}(t) \\
\mathbf{C}_{3}^{F}(t)
\end{array}\right), \\
\mathbf{H}^{F} & =\left(\begin{array}{ccc}
\mathbf{H}_{1} & 0 & 0 \\
0 & \mathbf{H}_{2} & 0 \\
0 & 0 & \mathbf{H}_{3}
\end{array}\right)+\left(\begin{array}{ccc}
0 & \mathbf{V}_{12}^{F} & \mathbf{V}_{13}^{F} \\
\mathbf{V}_{21}^{F} & 0 & \mathbf{V}_{23}^{F} \\
\mathbf{V}_{31}^{F} & \mathbf{V}_{32}^{F} & 0
\end{array}\right),
\end{aligned}
$$

and

$$
\boldsymbol{\Delta}=\left(\begin{array}{ccc}
\boldsymbol{\Delta}_{1} & 0 & 0 \\
0 & \boldsymbol{\Delta}_{2} & 0 \\
0 & 0 & \boldsymbol{\Delta}_{3}
\end{array}\right)
$$

one can write the discretized TDFE in the compact matrix form

$$
i \Delta \frac{d \mathbf{C}^{F}(t)}{d t}=\mathbf{H}^{F} \mathbf{C}^{F}(t) .
$$

To solve this system of first-order differential equations, the central difference approximation of the time derivative provides a convenient propagation scheme:

$$
\mathbf{C}^{F}\left(t_{n+1}\right)=-2 i(\delta t) \boldsymbol{\Delta}^{-1} \mathbf{H}^{F} \mathbf{C}^{F}\left(t_{n}\right)+\mathbf{C}^{F}\left(t_{n-1}\right),
$$

where $\delta t$ is the time step, and $t_{n}=n \delta t$. More sophisticated time-propagation schemes could be employed, but this conditionally stable scheme has proven to be satisfactory for 
present purposes. The presence of the $\boldsymbol{\Delta}^{-1}$ matrix in Eq. (41) is rather innocuous because $\boldsymbol{\Delta}$ has a block-diagonal structure in rearrangement indices and each block $\boldsymbol{\Delta}_{\beta}$ is a direct product of two smaller matrices, namely, $\boldsymbol{\Delta}_{\beta}=\boldsymbol{\Delta}_{\beta p} \otimes \boldsymbol{\Delta}_{\beta q}$. This is to be contrasted with the nontrivial task of inverting the non-orthogonality matrix appearing in the CRC approach of Eq. (16).

\section{Discretizaton of spatial variables and time in TDTFE}

Structure of TDTFE is also well suited to the simultaneous use of all three rearrangement bases. Each $\Psi_{\beta}^{T F}(t), \beta=$ $1,2,3$, is expanded in its natural separable basis $\left\{\left|u_{i} v_{j}\right\rangle_{\beta}\right\}$, viz.,

$$
\left|\Psi_{\beta}^{T F}(t)\right\rangle=\sum_{i=1}^{I_{\beta}} \sum_{j=1}^{J_{\beta}}\left|u_{i} v_{j}\right\rangle_{\beta} c_{\beta i j}^{T F}(t) .
$$

The initial condition (26) becomes

$$
c_{\beta i j}^{T F}(0)=\sum_{i^{\prime}=1}^{I_{\beta}} \sum_{j^{\prime}=1}^{J_{\beta}}\left(\boldsymbol{\Delta}_{\beta}^{-1}\right)_{i j, i^{\prime} j^{\prime}}{ }_{\beta}\left\langle u_{i^{\prime}} v_{j^{\prime}} \mid \Phi_{\alpha n_{0} q_{0}}(0)\right\rangle .
$$

Applying the Galerkin scheme one obtains

$$
\boldsymbol{\Delta}_{\alpha} \frac{d \mathbf{C}_{\alpha}^{T F}(t)}{d t}=\mathbf{H}_{\alpha} \mathbf{C}_{\alpha}^{T F}(t)+\sum_{\beta} \bar{\delta}_{\alpha \beta} \mathbf{V}_{\alpha \beta}^{T F} \mathbf{C}_{\beta}^{T F}(t),
$$

where $\mathbf{C}_{\alpha}^{T F}(t)$ is the column vector of expansion coefficients $c_{\alpha i j}^{T F}(t)$, and

$$
\left(\mathbf{V}_{\alpha \beta}^{T F}\right)_{i j, i^{\prime} j^{\prime}}={ }_{\alpha}\left\langle u_{i} v_{j}\left|V_{\beta}\right| u_{i^{\prime}} v_{j^{\prime}}\right\rangle_{\beta}
$$

By defining

$$
\mathbf{C}^{T F}(t)=\left(\begin{array}{l}
\mathbf{C}_{1}^{T F}(t) \\
\mathbf{C}_{2}^{T F}(t) \\
\mathbf{C}_{3}^{T F}(t)
\end{array}\right)
$$

and

$$
\mathbf{H}^{T F}=\left(\begin{array}{ccc}
\mathbf{H}_{1} & 0 & 0 \\
0 & \mathbf{H}_{2} & 0 \\
0 & 0 & \mathbf{H}_{3}
\end{array}\right)+\left(\begin{array}{ccc}
0 & \mathbf{V}_{12}^{T F} & \mathbf{V}_{13}^{T F} \\
\mathbf{V}_{21}^{T F} & 0 & \mathbf{V}_{23}^{T F} \\
\mathbf{V}_{31}^{T F} & \mathbf{V}_{32}^{T F} & 0
\end{array}\right),
$$

one can write the discretized TDTFE in the compact matrix form

$$
i \Delta \frac{d \mathbf{C}^{T F}(t)}{d t}=\mathbf{H}^{T F} \mathbf{C}^{T F}(t) .
$$

Using the central difference approximation for the time derivative, one obtains the TDTFE version of Eq. (41) as

$$
\mathbf{C}^{T F}\left(t_{n+1}\right)=-2 i(\delta t) \boldsymbol{\Delta}^{-1} \mathbf{H}^{T F} \mathbf{C}^{T F}\left(t_{n}\right)+\mathbf{C}^{T F}\left(t_{n-1}\right) \text {. }
$$

\section{IMPLEMENTATION OF PERMUTATIONAL SYMMETRY}

One can proceed in two ways to incorporate the effects of particle identity.

(i) Postsymmetrization. The calculation is carried out as if identical particles are distinguishable, and the resulting distinguishable $S$ matrices are combined to obtain the physical $S$ matrices (symmetrized or antisymmetrized as the case may be).

(ii) Prior symmetrization. The permutational symmetry is incorporated into the Faddeev equations. As is well known from the TI context, when one has three identical particles, three Faddeev equations can be reduced to a single Faddeev equation. Of course, similar reduction occurs in the time-dependent case. Thus the prior symmetrization has the advantage of involving less computational work.

Both schemes have been used in the present study, partly to ensure the reliability of numerical procedures and coding practices. Instead of going over the full machinery of postand prior-symmetrization schemes for the general three-body system, this section specializes to the model problem of three identical particles involving only $s$-wave two-body interactions and zero-angular momentum states. The two special cases that will be considered are (i) three identical spin-0 bosons, (ii) quartet spin state of three nucleons.

\section{A. Permutational symmetry for Faddeev equations}

\section{Three identical spinless particles}

If one just proceeds as if identical particles are distinguishable, one first notes that, for collisions starting in rearrangement 1 , the expansion coefficients satisfy $\mathbf{C}_{2}^{F}=\mathbf{C}_{3}^{F}$ in the discretized TDFE. Here it is assumed that all particles are treated on equal footing. In particular, all three rearrangement bases are identical. With this caveat in mind, for our model system one has $\mathbf{H}_{1}=\mathbf{H}_{2}=\mathbf{H}_{3}, \boldsymbol{\Delta}_{1}=\boldsymbol{\Delta}_{2}=\boldsymbol{\Delta}_{3}$, and $\mathbf{V}_{12}^{F}=$ $\mathbf{V}_{21}^{F}=\mathbf{V}_{13}^{F}=\mathbf{V}_{31}^{F}=\mathbf{V}_{23}^{F}=\mathbf{V}_{32}^{F}$. Hence, three equations for the component vectors reduces to two coupled equations, viz.,

$$
\begin{aligned}
& i \boldsymbol{\Delta}_{1} \frac{d \mathbf{C}_{1}^{F}(t)}{d t}=\mathbf{H}_{1} \mathbf{C}_{1}^{F}(t)+2 \mathbf{V}_{12}^{F} \mathbf{C}_{2}^{F}(t) \\
& i \boldsymbol{\Delta}_{1} \frac{d \mathbf{C}_{2}^{F}(t)}{d t}=\mathbf{H}_{1} \mathbf{C}_{2}^{F}(t)+\mathbf{V}_{12}^{F}\left[\mathbf{C}_{1}^{F}(t)+\mathbf{C}_{2}^{F}(t)\right]
\end{aligned}
$$

After numerical propagation of vectors $\mathbf{C}_{1}^{F}(t)$ and $\mathbf{C}_{2}^{F}(t)$ to asymptotic times, one can construct the components $\left|\psi_{1}^{F}(t)\right\rangle$, and $\left|\psi_{2}^{F}(t)\right\rangle$, from which distinguishable-particle $S$ matrices $\hat{S}_{1 n, 1 n_{0}}$ and $\hat{S}_{2 n, 1 n_{0}}$ are obtained using either Eq. (30) or Eq. (14). Note that, for the model considered, $\hat{S}_{2 n, 1 n_{0}}=\hat{S}_{3 n, 1 n_{0}}$. The symmetrized $S$ matrix is then given by

$$
\hat{S}_{n n_{0}}^{\mathrm{sym}}=\hat{S}_{1 n, 1 n_{0}}+2 \hat{S}_{2 n, 1 n_{0}} .
$$

For the determination of $\hat{S}_{n n_{0}}^{\text {sym }}$, one does not really need to solve for the individual vectors $\mathbf{C}_{1}^{F}(t), \mathbf{C}_{2}^{F}(t)$, and $\mathbf{C}_{3}^{F}(t)$. It would suffice to solve for their symmetric combination $\mathbf{C}_{\text {sym }}^{F}(t)=\mathbf{C}_{1}^{F}(t)+\mathbf{C}_{2}^{F}(t)+\mathbf{C}_{3}^{F}(t)=\mathbf{C}_{1}^{F}(t)+2 \mathbf{C}_{2}^{F}(t)$. Combining Eqs. (50) and (51), one finds

$$
i \boldsymbol{\Delta}_{1} \frac{d \mathbf{C}_{\mathrm{sym}}^{F}(t)}{d t}=\left[\mathbf{H}_{1}+2 \mathbf{V}_{12}^{F}\right] \mathbf{C}_{\mathrm{sym}}^{F}(t) \text {. }
$$

The initial condition is $\mathbf{C}_{\text {sym }}^{F}(0)=\mathbf{C}_{1}^{F}(0)$. It can be noted in passing that Eq. (53) is the discretized version of the prior- 
symmetrized Faddeev equation, viz.,

$$
i \frac{\partial\left|\psi_{\mathrm{sym}}^{F}(t)\right\rangle}{\partial t}=\left[H_{1}+V_{1}\left(P_{123}+P_{132}\right)\right]\left|\psi_{\mathrm{sym}}^{F}(t)\right\rangle,
$$

where $P_{123}$ and $P_{132}$ are the even permutation operators.

In the computational implementation of the priorsymmetrization scheme, $\mathbf{C}_{\text {sym }}(t)$ is obtained by solving Eq. (53) and the symmetrized Faddeev component $\left|\psi_{\text {sym }}^{F}(t)\right\rangle$ is constructed using $\left|\psi_{\text {sym }}^{F}(t)\right\rangle=\sum_{i} \sum_{j}\left|u_{i} v_{j}\right\rangle_{1}\left[\mathbf{C}_{\text {sym }}^{F}(t)\right]_{i j}$. The symmetrized $S$ matrix $\hat{S}_{n n_{0}}^{\text {sym }}$ follows from the analysis of $\left|\psi_{\text {sym }}^{F}(t)\right\rangle$ at asymptotic times:

$$
\hat{S}_{n^{\prime} q^{\prime}, n_{0} q}^{\text {sym }}(E)=\sqrt{\frac{q^{\prime}}{q}} \frac{{ }_{1}\left\langle\phi_{n^{\prime}} q^{\prime} \mid \psi_{\text {sym }}^{F}(t)\right\rangle}{{ }_{1}\left\langle\phi_{n_{0}} q \mid \Phi_{1 n_{0} q_{0}}(t)\right\rangle} .
$$

Alternatively one can form the symmetrized full wave packet

$$
\begin{aligned}
\left|\Psi_{\mathrm{sym}}(t)\right\rangle & =\left(I+P_{123}+P_{132}\right)\left|\psi_{\mathrm{sym}}^{F}(t)\right\rangle \\
& =\left(I+P_{123}+P_{132}\right) \sum_{i} \sum_{j}\left|u_{i} v_{j}\right\rangle_{1}\left[\mathbf{C}_{\mathrm{sym}}^{F}(t)\right]_{i j},
\end{aligned}
$$

and extract the elastic $S$ matrix via

$$
\hat{S}_{n^{\prime} q^{\prime}, n_{0} q}^{\mathrm{sym}}(E)=\sqrt{\frac{q^{\prime}}{q}} \frac{{ }_{1}\left\langle\phi_{n^{\prime}} q^{\prime} \mid \Psi_{\text {sym }}(t)\right\rangle}{{ }_{1}\left\langle\phi_{n_{0}} q \mid \Phi_{1 n_{0} q_{0}}(t)\right\rangle} .
$$

\section{Three nucleons in quartet spin state}

One can apply a similar procedure to implement the antisymmetrization requirement for the system of three identical spin- $1 / 2$ fermions. For the special case of the quartet state of three nucleons (within $s$-wave interaction model and for zero angular momentum states), the antisymmetrized $S$-matrix elements is obtained from the distinguishable-particle $S$-matrix elements via

$$
\hat{S}_{n n_{0}}^{\text {anti }}=\hat{S}_{1 n, 1 n_{0}}-0.5 \hat{S}_{2 n, 1 n_{0}}-0.5 \hat{S}_{3 n, 1 n_{0}}=\hat{S}_{1 n, 1 n_{0}}-\hat{S}_{2 n, 1 n_{0}} .
$$

The factor of -0.5 in this equation represents the spin-isospin recoupling coefficient between rearrangements of three nucleons in the quartet spin state.

If one is interested in the determination of $\hat{S}_{n n_{0}}^{\text {anti }}$ only, it would suffice to solve for the antisymmetric combination $\mathbf{C}_{\text {anti }}^{F}(t)=\mathbf{C}_{1}^{F}(t)-0.5 \mathbf{C}_{2}^{F}(t)-0.5 \mathbf{C}_{3}^{F}(t)=\mathbf{C}_{1}^{F}(t)-$ $\mathbf{C}_{2}^{F}(t)$. The time-evolution equation for $\mathbf{C}_{\text {anti }}^{F}(t)$ is obtained by combining Eqs. (50) and (51):

$$
i \boldsymbol{\Delta}_{1} \frac{d \mathbf{C}_{\text {anti }}^{F}(t)}{d t}=\left[\mathbf{H}_{1}-\mathbf{V}_{12}^{F}\right] \mathbf{C}_{\text {anti }}^{F}(t) .
$$

Again this equation is the discretized version of the priorantisymmetrized Faddeev equation:

$$
i \frac{\partial\left|\psi_{\text {anti }}^{F}(t)\right\rangle}{\partial t}=\left[H_{1}+V_{1}\left(P_{123}+P_{132}\right)\right]\left|\psi_{\text {anti }}^{F}(t)\right\rangle,
$$

As before the incorporation of the antisymmetry requirement reduces the computational burden. In the computational implementation of this prior-antisymmetrization scheme, $\mathbf{C}_{\text {anti }}^{F}(t)$ is obtained by solving Eq. (59) and the antisymmetrized Faddeev component $\left|\psi_{\text {anti }}^{F}(t)\right\rangle$ is constructed using $\left|\psi_{\text {anti }}^{F}(t)\right\rangle=\sum_{i} \sum_{j}\left|u_{i} v_{j}\right\rangle_{1}\left(\mathbf{C}_{\text {anti }}^{F}(t)\right)_{i j}$. The antisymmetrized $S$-matrix element $\hat{S}_{n n_{0}}^{\text {anti }}$ is then obtained from the asymptotic analysis of $\left|\psi_{\text {anti }}^{F}(t)\right\rangle$ via

$$
\hat{S}_{n^{\prime} q^{\prime}, n_{0} q}^{\mathrm{anti}}(E)=\sqrt{\frac{q^{\prime}}{q}} \frac{{ }_{1}\left\langle\phi_{n^{\prime}} q^{\prime} \mid \psi_{\mathrm{anti}}^{F}(t)\right\rangle}{{ }_{1}\left\langle\phi_{n_{0}} q \mid \Phi_{1 n_{0} q_{0}}(t)\right\rangle} .
$$

Alternatively one can form the antisymmetrized full wave packet

$$
\begin{aligned}
\left|\Psi_{\text {anti }}^{F}(t)\right\rangle & =\left(I+P_{123}+P_{132}\right)\left|\psi_{\text {anti }}^{F}(t)\right\rangle \\
& =\left(I+P_{123}+P_{132}\right) \sum_{i} \sum_{j}\left|u_{i} v_{j}\right\rangle_{1}\left[\mathbf{C}_{\text {anti }}^{F}(t)\right]_{i j},
\end{aligned}
$$

and extract the elastic $S$ matrix via

$$
\hat{S}_{n^{\prime} q^{\prime}, n_{0} q}^{\mathrm{anti}}(E)=\sqrt{\frac{q^{\prime}}{q}} \frac{{ }_{1}\left\langle\phi_{n^{\prime}} q^{\prime} \mid \Psi_{\mathrm{anti}}^{F}(t)\right\rangle}{{ }_{1}\left\langle\phi_{n_{0}} q \mid \Phi_{1 n_{0} q_{0}}(t)\right\rangle} .
$$

\section{B. Permutational symmetry for transposed Faddeev equations}

Introduction of permutational symmetry into the transposed Faddeev equations proceed pretty much like that of the Faddeev equations.

\section{Three identical spinless particles}

Since for three identical particles in the present model, $\mathbf{H}_{1}=\mathbf{H}_{2}=\mathbf{H}_{3}, \boldsymbol{\Delta}_{1}=\boldsymbol{\Delta}_{2}=\boldsymbol{\Delta}_{3}$, and $\mathbf{V}_{12}^{T F}=\mathbf{V}_{21}^{T F}=\mathbf{V}_{13}^{T F}=$ $\mathbf{V}_{31}^{T F}=\mathbf{V}_{23}^{T F}=\mathbf{V}_{32}^{T F}$, the three equations for $\mathbf{C}_{1}^{T F}, \mathbf{C}_{2}^{T F}$, and $\mathbf{C}_{3}^{T F}$ reduce to two coupled equations, viz.,

$$
\begin{aligned}
& i \Delta_{1} \frac{d \mathbf{C}_{1}^{T F}(t)}{d t}=\mathbf{H}_{1} \mathbf{C}_{1}^{T F}(t)+2 \mathbf{V}_{12}^{T F} \mathbf{C}_{2}^{T F}(t) \\
& i \Delta_{1} \frac{d \mathbf{C}_{2}^{T F}(t)}{d t}=\mathbf{H}_{1} \mathbf{C}_{2}^{T F}(t)+\mathbf{V}_{12}^{T F}\left[\mathbf{C}_{1}^{T F}(t)+\mathbf{C}_{2}^{T F}(t)\right],
\end{aligned}
$$

where the fact that $\mathbf{C}_{2}^{T F}=\mathbf{C}_{3}^{T F}$ has been used. After numerical propagation of vectors $\mathbf{C}_{1}^{T F}(t)$ and $\mathbf{C}_{2}^{T F}(t)$ to asymptotic times, one can construct the states $\left|\Psi_{1}^{T F}(t)\right\rangle$, and $\left|\Psi_{2}^{T F}(t)\right\rangle$, from which distinguishable-particle $S$ matrices $\hat{S}_{1 n, 1 n_{0}}$ and $\hat{S}_{2 n, 1 n_{0}}$ are obtained using Eq. (30). The symmetrized $S$ matrix $\hat{S}_{n n_{0}}^{\text {sym }}$ is then given by Eq. (52).

For the determination of $\hat{S}_{n n_{0}}^{\text {sym }}$, it would suffice to solve for the symmetric combination $\mathbf{C}_{\text {sym }}^{T F}(t)=\mathbf{C}_{1}^{T F}(t)+\mathbf{C}_{2}^{T F}(t)+$ $\mathbf{C}_{3}^{T F}(t)=\mathbf{C}_{1}^{T F}(t)+2 \mathbf{C}_{2}^{T F}(t)$. Combining Eqs. (64) and (65), one finds

$$
i \boldsymbol{\Delta}_{1} \frac{d \mathbf{C}_{\mathrm{sym}}^{T F}(t)}{d t}=\left[\mathbf{H}_{1}+2 \mathbf{V}_{12}^{F}\right] \mathbf{C}_{\mathrm{sym}}^{T F}(t) .
$$

The initial condition becomes $\mathbf{C}_{\text {sym }}^{T F}(0)=\mathbf{C}_{1}^{T F}(0)+$ $\mathbf{C}_{2}^{T F}(0)+\mathbf{C}_{3}^{T F}(0)$. In passing it is noted that Eq. (66) is the discretized version of the prior-symmetrized form of the transposed Faddeev equation, viz.,

$$
i \frac{\partial\left|\Psi_{\mathrm{sym}}^{T F}(t)\right\rangle}{\partial t}=\left[H_{1}+\left(P_{123}+P_{132}\right)\right] V_{1}\left|\Psi_{\mathrm{sym}}^{T F}(t)\right\rangle .
$$


In the computational implementation of the priorsymmetrization scheme, $\mathbf{C}_{\mathrm{sym}}^{T F}(t)$ is obtained by solving Eq. (66) and the symmetrized state $\left|\Psi_{\text {sym }}^{T F}(t)\right\rangle$ is constructed using $\left|\Psi_{\text {sym }}^{T F}(t)\right\rangle=\sum_{i} \sum_{j}\left|u_{i} v_{j}\right\rangle_{1}\left[\mathbf{C}_{\text {sym }}^{T F}(t)\right]_{i j}$. The symmetrized $S$ matrix $\hat{S}_{n n_{0}}^{\text {sym }}$ then follows from the analysis of $\left|\Psi_{\text {sym }}^{T F}(t)\right\rangle$ at asymptotic times:

$$
\hat{S}_{n^{\prime} q^{\prime}, n_{0} q}^{\mathrm{sm}}(E)=\sqrt{\frac{q^{\prime}}{q}} \frac{{ }_{1}\left\langle\phi_{n^{\prime}} q^{\prime} \mid \Psi_{\mathrm{sym}}^{T F}(t)\right\rangle}{{ }_{1}\left\langle\phi_{n_{0}} q \mid \Phi_{1 n_{0} q_{0}}(t)\right\rangle} .
$$

\section{Three nucleons in quartet spin state}

As in the case for Faddeev equations, the antisymmetrized $S$-matrix elements is obtained from the distinguishableparticle $S$-matrix elements via Eq. (58). If one is solely interested in the determination of $\hat{S}_{n n_{0}}^{\text {anti }}$, it would suffice to solve for the antisymmetric combination $\mathbf{C}_{\text {anti }}^{T F}(t)=$ $\mathbf{C}_{1}^{T F}(t)-0.5 \mathbf{C}_{2}^{T F}(t)-0.5 \mathbf{C}_{3}^{T F}(t)=\mathbf{C}_{1}^{T F}(t)-\mathbf{C}_{2}^{T F}(t)$. The time-evolution equation for $\mathbf{C}_{\text {anti }}^{T F}(t)$ is obtained by combining Eqs. (64) and (65):

$$
i \boldsymbol{\Delta}_{1} \frac{d \mathbf{C}_{\mathrm{anti}}^{T F}(t)}{d t}=\left[\mathbf{H}_{1}-\mathbf{V}_{12}^{T F}\right] \mathbf{C}_{\mathrm{anti}}^{T F}(t) .
$$

Again, the incorporation of the antisymmetry requirement reduces the computational burden. In the computational implementation of this postantisymmetrization scheme, $\mathbf{C}_{\text {anti }}^{T F}(t)$ is obtained by solving Eq. (69) and the antisymmetrized wave packet $\left|\Psi_{\text {anti }}^{T F}(t)\right\rangle$ is constructed using $\left|\Psi_{\text {anti }}^{T F}(t)\right\rangle=$ $\sum_{i} \sum_{j}\left|u_{i} v_{j}\right\rangle_{1}\left(\mathbf{C}_{\text {anti }}^{T F}(t)\right)_{i j}$. The antisymmetrized $S$-matrix element $\hat{S}_{n n_{0}}^{\text {anti }}$ is then obtained from the asymptotic analysis of $\left|\Psi_{\text {anti }}^{T F}(t)\right\rangle$ via

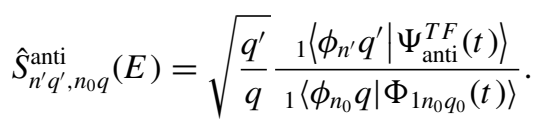

\section{Vi. CALCUlations AND RESUlTS}

\section{A. Reference results}

Reference results for the three-boson and three-fermion models described in Sec. V has been obtained within timeindependent framework. For the separable pair potential, momentum-space Faddeev integral equations for rearrangement transition operators has been used. As is well known, with separable pair potentials, Faddeev equations are reduced to a set of effective two-body integral equations in the spectator momenta. However, above the breakup threshold, the effective potentials of these integral equations have logarithmic singularities that require careful treatment. For the present study, the effective two-body equations have been solved using Schwinger variational principle with a basis of piecewise polynomials in the spectator momentum. Due attention has been paid to the logarithmic singularities in the effective potential term in accordance with the analysis of singularities given in Ref. [28]. A subtraction scheme similar to the one used in Ref. [29] has been devised to calculate matrix elements involving these singularities.

Reference results for the three-boson and three-fermion models with the local pair potential (MT-III) has been obtained using an extension of the coupled-reaction-channels
TABLE I. Inelasticity parameter $|S|$ and real part of phase shift $\delta_{R}$ for the seperable potential model. Results obtained from the numerical wave-packet solutions of TDTFE are compared with the results of two different sets of time-independent calculations. Faddeev results refer to Faddeev integral-equation calculations in momentum space, while CRC refers to the calculations using the CRC ansatz supplemented with two-body pseudostates. Results obtained from the wave packet at different asymptotic times $T$ are listed for various values of spectator momentum $q$. Wave-packet parameters are $q_{0}=2 \mathrm{fm}^{-1}$, $y_{0}=9 \mathrm{fm}, d=1.5 \mathrm{fm}$. Finite-element grid corresponds to $\mathcal{I}_{p}=44$ and $\mathcal{J}_{q}=201$.

\begin{tabular}{lccccc}
\hline \hline$q\left(\mathrm{fm}^{-2}\right)$ & $T\left(\mathrm{fm}^{2}\right)$ & $\left|\hat{S}^{\text {sym }}\right|$ & $\delta_{R}^{\text {sym }}$ & $\left|\hat{S}^{\text {anti }}\right|$ & $\delta_{R}^{\text {anti }}$ \\
\hline 0.50 & 32 & 0.8462 & 171.59 & 1.0347 & 77.39 \\
& 40 & 0.8581 & 170.50 & 1.0119 & 78.47 \\
& 48 & 0.8844 & 170.26 & 0.9889 & 78.22 \\
& CRC & 0.8959 & 172.42 & 0.9876 & 77.59 \\
& Faddeev & 0.8959 & 172.35 & 0.9876 & 77.51 \\
1.00 & 32 & 0.4113 & 119.54 & 0.8827 & 39.90 \\
& 40 & 0.4148 & 119.25 & 0.8829 & 39.91 \\
& 48 & 0.4126 & 119.32 & 0.8835 & 39.91 \\
& CRC & 0.4176 & 119.25 & 0.8824 & 40.01 \\
& Faddeev & 0.4183 & 119.11 & 0.8825 & 39.96 \\
1.50 & 24 & 0.3178 & 52.68 & 0.9068 & 19.16 \\
& 32 & 0.3203 & 52.71 & 0.9073 & 19.16 \\
& 40 & 0.3223 & 52.97 & 0.9081 & 19.17 \\
& 48 & 0.3230 & 53.21 & 0.9089 & 19.18 \\
& CRC & 0.3177 & 52.97 & 0.9065 & 19.19 \\
& Faddeev & 0.3181 & 53.05 & 0.9069 & 19.15 \\
2.00 & 24 & 0.6006 & 26.32 & 0.9541 & 9.38 \\
& 32 & 0.6010 & 26.29 & 0.9545 & 9.38 \\
& 40 & 0.6006 & 26.30 & 0.9549 & 9.38 \\
& 48 & 0.6010 & 26.29 & 0.9555 & 9.38 \\
& CRC & 0.5966 & 26.34 & 0.9535 & 9.39 \\
& Faddeev & 0.5974 & 26.33 & 0.9537 & 9.37 \\
& 24 & 0.7871 & 15.49 & 0.9773 & 4.82 \\
& 32 & 0.7874 & 15.51 & 0.9795 & 4.82 \\
& 40 & 0.7876 & 15.55 & 0.9797 & 4.83 \\
& 48 & 0.7884 & 15.56 & 0.9800 & 4.83 \\
& Faddeev & 0.7846 & 15.56 & 0.9791 & 4.81 \\
\hline \hline & & & & & \\
& & 0.7837 & 15.64 & 0.9790 & 4.82 \\
& & & & & \\
& & & & \\
& & &
\end{tabular}

(CRC) method. In this approach, the usual CRC expansion ansatz is supplemented with pseudostates in each rearrangement as described in Ref. [24]. In effect, the two-body continuum is simulated by a discrete set of pseudostates. To ensure convergence for a rather wide range of collision energies, the reference CRC results quoted in this paper were obtained using a set of 80 pseudostates for each two-particle subsystem. A comparison of the results from Faddeev and CRC approaches for the separable potential model are given in Table I for selected values of $q$. That this approach is capable of producing accurate results for the MT-III model with a much smaller pseudostate basis has already been demonstrated in Ref. [25]. 
TABLE II. Inelasticity parameter $|S|$ and real part of phase shift $\delta_{R}$ for the local potential model. Results obtained from the numerical wave-packet solutions of TDTFE are compared with the $\mathrm{CRC}$ results at a number of collision energies. Benchmark results from the literature are also given for two of the collision energies. Results obtained from the wave packet at different asymptotic times $T$ are listed for various values of spectator laboratory energy $E_{\text {in }}^{\mathrm{Lab}}$. Wave-packet parameters are $q_{0}=2 \mathrm{fm}^{-1}, y_{0}=9 \mathrm{fm}, d=1.5 \mathrm{fm}$. Finite-element grid corresponds to $\mathcal{I}_{p}=44$ and $\mathcal{J}_{q}=201$.

\begin{tabular}{|c|c|c|c|c|c|c|}
\hline $\begin{array}{l}\left.E_{\mathrm{in}}^{\mathrm{Lab}}\right) \\
(\mathrm{MeV})\end{array}$ & $q\left(\mathrm{fm}^{-2}\right)$ & $T\left(\mathrm{fm}^{2}\right)$ & $\left|\hat{S}^{\mathrm{sym}}\right|$ & $\delta_{R}^{\mathrm{sym}}$ & $\left|\hat{S}^{\text {anti }}\right|$ & $\delta_{R}^{\text {anti }}$ \\
\hline \multirow[t]{5}{*}{14.1} & \multirow[t]{5}{*}{0.5498} & 32 & 0.7918 & 152.23 & 1.0083 & 69.68 \\
\hline & & 40 & 0.8088 & 152.93 & 0.9756 & 69.64 \\
\hline & & 48 & 0.8279 & 152.71 & 0.9787 & 69.18 \\
\hline & & $\mathrm{CRC}$ & 0.8302 & 153.12 & 0.9783 & 68.96 \\
\hline & & Refs. [22,23] & - & - & 0.9782 & 68.95 \\
\hline \multirow[t]{5}{*}{42.0} & \multirow[t]{5}{*}{0.9488} & 32 & 0.4333 & 105.77 & 0.9009 & 37.58 \\
\hline & & 40 & 0.4362 & 105.74 & 0.9019 & 37.60 \\
\hline & & 48 & 0.4417 & 104.63 & 0.9029 & 37.60 \\
\hline & & CRC & 0.4328 & 105.21 & 0.9033 & 37.71 \\
\hline & & Refs. [22,23] & - & - & 0.9035 & 37.71 \\
\hline \multirow[t]{5}{*}{105.0} & \multirow[t]{5}{*}{1.50} & 24 & 0.4997 & 38.32 & 0.9459 & 13.90 \\
\hline & & 32 & 0.5037 & 38.37 & 0.9465 & 13.90 \\
\hline & & 40 & 0.5046 & 38.47 & 0.9471 & 13.90 \\
\hline & & 48 & 0.5045 & 38.65 & 0.9479 & 13.91 \\
\hline & & CRC & 0.5009 & 38.46 & 0.9452 & 13.6 \\
\hline \multirow[t]{5}{*}{186.6} & \multirow[t]{5}{*}{2.00} & 24 & 0.7903 & 16.58 & 0.9863 & 4.91 \\
\hline & & 32 & 0.7910 & 16.59 & 0.9867 & 4.91 \\
\hline & & 40 & 0.7912 & 16.59 & 0.9872 & 4.91 \\
\hline & & 48 & 0.7919 & 16.60 & 0.9876 & 4.92 \\
\hline & & $\mathrm{CRC}$ & 0.7880 & 16.63 & 0.9858 & 4.95 \\
\hline \multirow[t]{5}{*}{291.6} & \multirow[t]{5}{*}{2.50} & 24 & 0.9431 & 6.03 & 0.9791 & 1.17 \\
\hline & & 32 & 0.9438 & 6.04 & 0.9981 & 1.17 \\
\hline & & 40 & 0.9446 & 6.06 & 0.9984 & 1.17 \\
\hline & & 48 & 0.9452 & 6.06 & 0.9985 & 1.18 \\
\hline & & CRC & 0.9424 & 6.07 & 0.9976 & 1.18 \\
\hline
\end{tabular}

For the fermion model with MT-III potential, Table II contains a comparison of CRC results with benchmark results from Refs. [22,23] at two collision energies. Unfortunately, such benchmark results for the boson case are not available and the $\mathrm{CRC}$ results alone are used as the reference.

Since in the three-particle model under consideration there is only one bound state in each rearrangement, the only rearrangement $S$ matrices occurring after proper account of permutational symmetry are $\hat{S}_{1 q, 1 q}^{\mathrm{sym}}(E)$ and $\hat{S}_{1 q, 1 q}^{\text {anti }}(E)$, which correspond to the symmetrized and antisymmetrized rearrangement $S$-matrix elements of the three-boson and threefermion cases, respectively. In the presentation of results, these $S$-matrix elements are referred to as elastic $S$ matrices and are denoted as $\hat{S}^{\mathrm{sym}}(E)$ and $\hat{S}^{\mathrm{anti}}(E)$. Recall that $E$ and $q$ are related in the present model by $E=\epsilon+3 q^{2} / 4$. Here $\epsilon$ is the energy of the sole bound state of the two-particle system.

\section{B. Computational implementation of the wave-packet method}

For the expansion bases $u_{i}$ and $v_{j}$, piecewise quadratic polynomials [30] are used. For this purpose, a computational domain in the $p-q$ plane for each rearrangement is chosen by introducing the cutoff values $p_{\max }$ and $q_{\max }$. The values for these cutoffs are chosen by considering the momentum-space support of the wave packet. The interval $\left[0, p_{\max }\right]$ for the variable $p$ is partitioned into $\mathcal{I}_{p}$ finite elements, and a set of $I$ local quadratic interpolation functions $\tilde{u}_{i}(p)$ are defined on this $p$ grid. Here $I=2 \mathcal{I}_{p}-1$. Similarly, $\left[0, q_{\max }\right]$ is partitioned into $\mathcal{J}_{q}$ finite elements, and $J$ local quadratic interpolation functions $\tilde{v}_{j}(q)$ are defined on the $q$ grid, with $J=2 \mathcal{J}_{q}-1$. Explicit forms of these local piecewise interpolation functions can be found in Ref. $[12,30]$. The basis functions $u_{i}(p)$ and $v_{j}(q)$ are then defined via

$$
u_{i}(p)=\frac{\tilde{u}_{i}(p)}{p} \quad \text { and } \quad u_{j}(q)=\frac{\tilde{v}_{j}(q)}{q} .
$$

Note that the finite-element grids do not have to be equally spaced, but are chosen to have a denser set of points in regions where wave packet is expected to have appreciable amplitude. For the $p$ variable we used $\mathcal{I}_{p}=44$ and the grid points were distributed as [0(10)0.5],[0.5(15)2.0],[2.0(5)3.0],[3.0(14)12], where the notation $[a(n) b]$ denotes the division of the interval $[a, b]$ into $n$ equal finite elements. For the $q$ variable, the finest grid used $\mathcal{J}_{q}=201$ finite elements. These choices give rise to $I=87$, and $J=401$, with the resulting dimension of the Hamiltonian matrix becoming 34887.

The finite-element grid for $q$ is chosen by taking into account the average and width of the momentum distribution of the initial wave packet. More points are used over the effective momentum support (defined for present purposes as the interval over which momentum probability density is greater than $1 \times 10^{-4}$ ) of the packet. Calculations reported in this paper employed two sets of parameters for the initial wave packet:

(i) $q_{0}=2 \mathrm{fm}^{-1}, y_{0}=9 \mathrm{fm}, d=1.5 \mathrm{fm}$. This wave packet is quite broad in momentum space: its effective momentum support is the interval $[0.0,4.0]$. For this case, the interval $\left[0, q_{\max }\right]$ was divided into 201 finite elements and the grid points were distributed as [0(30)0.3],[0.3(168)4.5],[4.5(3)4.8] where $[a(n) b]$ means that the interval $[a, b]$ is divided into $n$ equal finite elements.

(ii) $q_{0}=1 \mathrm{fm}^{-1}, y_{0}=18 \mathrm{fm}, d=3 \mathrm{fm}$. This packet is narrower in momentum space than the previous packet. Its effective momentum support is the interval $[0,2.0]$. Since this packet is wider in coordinate space, initial center of the packet is taken as $y_{0}=18$ in order to ensure that essentially almost all of the initial wave packet is outside the range of the interaction. The interval $\left[0, q_{\max }\right]$ was divided into 201 finite elements according to [0(100)1.0],[1.0(70)2.4],[2.4(31)4.8]. Another slightly less narrower momentum-space wave packet (with $q_{0}=1 \mathrm{fm}^{-1}, y_{0}=9, d=2.5 \mathrm{fm}$ ) was also used in some calculations. For the time propagation, the time step of $\delta t=0.005$ was found adequate. 


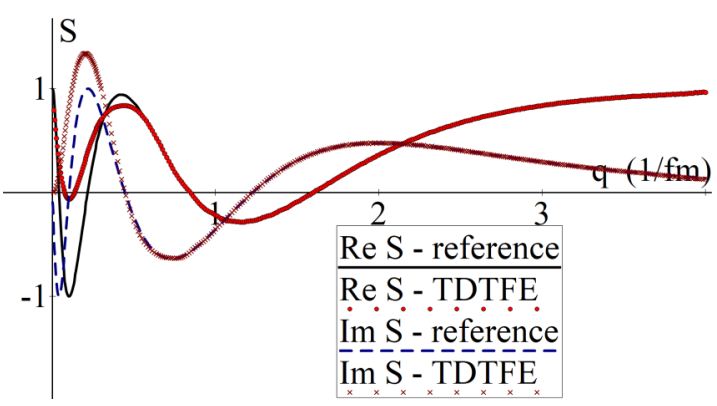

FIG. 1. Real and imaginary parts of the symmetrized $S$-matrix $S^{\text {sym }}$ for the three-boson model with separable pair potential are plotted as a function of spectator momentum $q$. Results obtained via Eq. (68) from the numerical wave-packet solution of the timedependent transposed Faddeev equation (TDTFE) are compared with reference results from solutions of time-independent momentumspace Faddeev integral equations. Parameters of the initial wave packet are $q_{0}=2 \mathrm{fm}^{-1}, y_{0}=9 \mathrm{fm}, d=1.5 \mathrm{fm}$.

$\hat{S}^{\text {sym }}(E)$ are extracted from the solutions of TDFE using asymptotic forms of either the Faddeev components via Eq. (55) or the total wave packet via Eq. (57). Similarly, $\hat{S}^{\text {anti }}(E)$ are extracted from asymptotic forms of the Faddeev components via Eq. (61) or the total wave packet via Eq. (63). On the other hand, solutions of TDTFE in asymptotic future yield $\hat{S}^{\text {sym }}(E)$ via Eq. (68) and $\hat{S}^{\text {anti }}(E)$ via Eq. (70).

\section{Results for three-boson and three-fermion models}

Figures 1 and 2 present the results of the time-dependent approach for the separable-potential model using the TDTFE version with the broader wave packet. Shown are $\hat{S}^{\mathrm{sym}}(E)$ and $\hat{S}^{\text {anti }}(E)$ extracted from numerically propagated total wave packet of the TDTFE approach at time $t=40 \mathrm{fm}^{2}$. For $q$ between $0.5 \mathrm{fm}^{-1}$ and $4 \mathrm{fm}^{-1}$, wave-packet results and reference results are essentially indistinguishable on these graphs. However, wave-packet results for low $q$ in the tail of the momentum distribution are of lower accuracy (fermion case) or even qualitatively wrong (boson case). For $q<0.5 \mathrm{fm}^{-1}$ where the momentum density of the initial wave packet is less than $5 \times 10^{-3}$, the results with this particular initial wave packet do not at all exhibit the dip that exists in reference

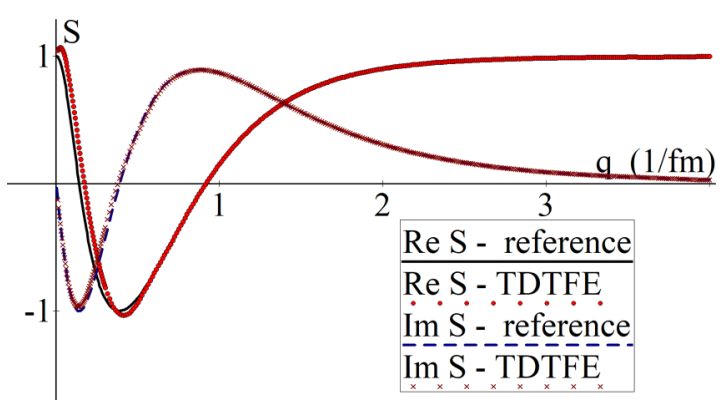

FIG. 2. Same as Fig. 1 but for $S^{\text {anti }}$ of the three-fermion model. Wave-packet results were calculated via Eq. (70) from the numerical wave-packet solution of the time-dependent transposed Faddeev equation (TDTFE).

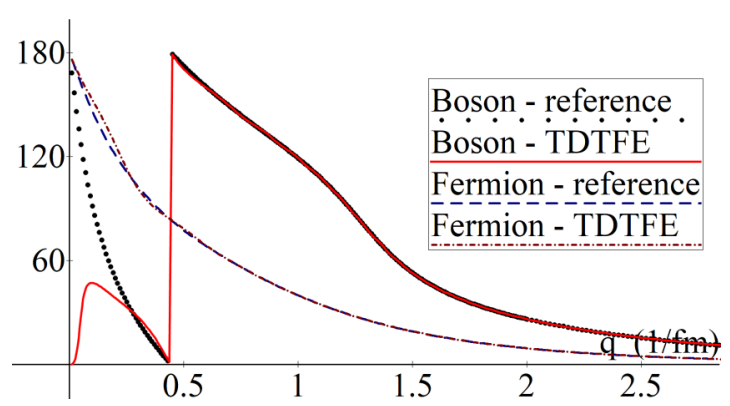

FIG. 3. Real part $\delta_{R}$ of phase shift for three-boson and threefermion models with separable pair potential. Results of wave packet calculation are compared with results from time-independent momentum-space Faddeev calculations. Parameters of the initial wave packet are $q_{0}=2 \mathrm{fm}^{-1}, y_{0}=9 \mathrm{fm}, d=1.5 \mathrm{fm}$.

results for $\operatorname{Im} \hat{S}^{\text {sym }}$. The dip in $\operatorname{Re} \hat{S}^{\text {sym }}$ is not fully recovered either. On the other hand, the results for the fermion model are more accurate in general and in the low-energy region as well.

It is customary to analyze such scattering information in terms of the inelasticity parameter $|\hat{S}|$ and the real part $\delta_{R}$ of the complex phase shift $\delta$ (defined via $\hat{S}=e^{2 i \delta}=|\hat{S}| e^{2 i \delta_{R}}$ ). Figures 3 and 4 show $|\hat{S}|$ and $\delta_{R}$ as a function of the spectator momentum $q$, respectively. The agreement between the wave-packet and Faddeev calculations is quite satisfactory for $q>0.5 \mathrm{fm}^{-1}$. Interestingly, $\delta_{R}$ for the boson case exhibits a jump of 180 degrees at about $q=0.445 \mathrm{fm}^{-1}$. In the low-energy end, for $q<0.5 \mathrm{fm}^{-1}$, inelasticity parameters from wave-packet calculations are seriously defective for both fermion and boson cases. Wave-packet results for $\delta_{R}$ in lowmomentum region have the wrong behavior for the boson case, while $\delta_{R}$ for the fermion case at least have the right type of dependence on $q$.

Note that at collision energies corresponding to $q<$ $0.2676 \mathrm{fm}^{-1}$ only elastic channel is open. For $q>$ $0.2676 \mathrm{fm}^{-1}$, the breakup channel is open and in fact dominates over rearrangement, especially, for the three-boson model. In Table I, a quantitative comparison of wave-packet results with reference results is made at selected values of $q$ and at various values of asymptotic time $T$. Although there is some variation with evaluation time $T$, the agreement between

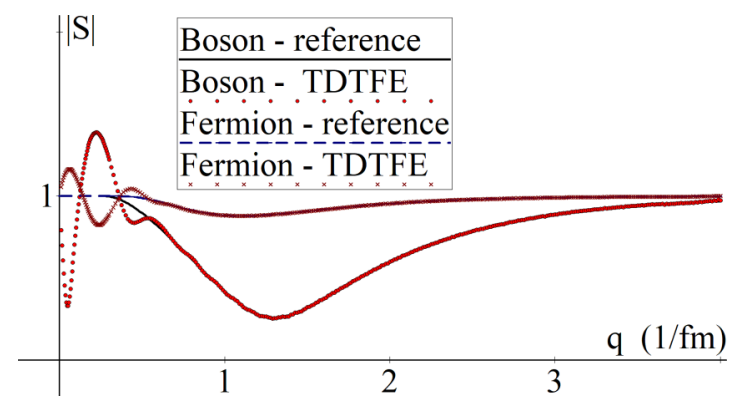

FIG. 4. Inelasticity parameter $|S|$ for three-boson and threefermion models with separable pair potential. Results of wavepacket calculation are compared with results from time-independent momentum-space Faddeev calculations. Parameters of the initial wave packet are $q_{0}=2 \mathrm{fm}^{-1}, y_{0}=9 \mathrm{fm}, d=1.5 \mathrm{fm}$. 


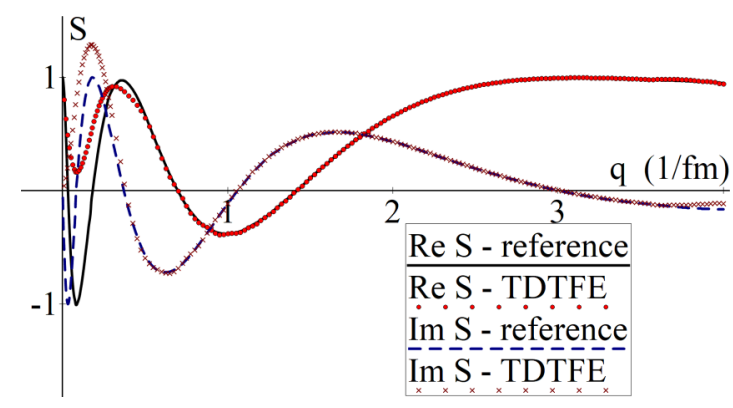

FIG. 5. Real and imaginary parts of the symmetrized $S$ matrix $S^{\text {sym }}$ for the three-boson model with the MT-III potential are plotted as a function of spectator momentum $q$. Parameters of the initial wave packet are $q_{0}=2 \mathrm{fm}^{-1}, y_{0}=9 \mathrm{fm}, d=1.5 \mathrm{fm}$.

the Faddeev and wave-packet results is quite satisfactory (except for $q=0.5 \mathrm{fm}^{-1}$ ) for both $|\hat{S}|$ and $\delta_{R}$. Thus, these calculations show that fairly accurate and stable rearrangement $S$ matrices can be extracted for over a rather large range of collision energies from a single wave-packet propagation.

Encouraged by these results, the time-dependent approach has next been tested on the model with local pair potential MT-III. Figures 5 and 6 give the $S$-matrix results obtained from the TDTFE approach with the same wave packet used in the separable-potential calculation reported above. In Figs. 7 and $8,|\hat{S}|$ and $\delta_{R}$ are given as functions of $q$. These graphs (Figs. 5-8) all look very much like the corresponding graphs (Figs. 1-4) of the separable potential model. The jump in $\delta_{R}$ is shifted to $q=0.375 \mathrm{fm}^{-1}$. Table II gives a quantitative comparison of wave-packet results (extracted at various values of asymptotic time $T$ ) with reference results at selected values of the energy $E_{\mathrm{in}}^{\mathrm{Lab}}$ of the incident particle in the laboratory frame. Note that, for $E_{\mathrm{in}}^{\mathrm{Lab}}=14.1 \mathrm{MeV}$ and $42 \mathrm{MeV}$, the accurate results that are available in the literature [22,23] are also shown for the fermion model with the MT-III pair potential. The same observations and comments made on the wave-packet results for the separable potential model apply to the MT-III potential case as well. Except for momentum components in the tails of the momentum space wave packet, $S$ matrices extracted from the asymptotic wave packet are reasonably accurate and stable.

For an initial wave packet with broad distribution in $q$, there is a great disparity between the passage times of highenergy and low-energy components. Long propagation times necessary for low-momentum components $q<0.5 \mathrm{fm}^{-1}$ may

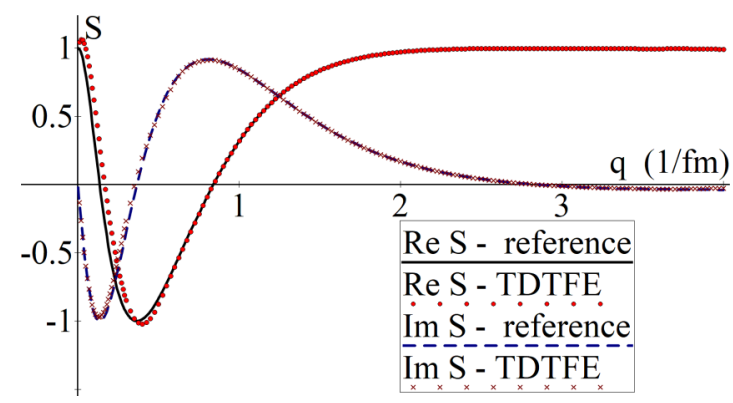

FIG. 6. Same as Fig. 5 but for $S^{\text {anti }}$ of the three-fermion model.

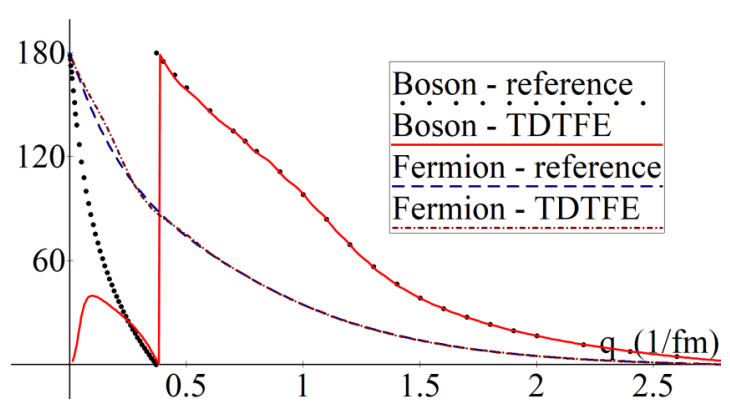

FIG. 7. Real part $\delta_{R}$ of phase shift for three-boson and threefermion models with the MT-III potential. Parameters of the initial wave packet are $q_{0}=2 \mathrm{fm}^{-1}, y_{0}=9 \mathrm{fm}, d=1.5 \mathrm{fm}$.

lead to boundary-reflection problem for high-momentum components. To avoid such complications, momentum distribution of the initial wave packet must be taken narrow. Accordingly, in an effort to improve the results for the lowenergy regime $\left(q<0.5 \mathrm{fm}^{-1}\right)$, an initial wave packet with $q_{0}=1.0 \mathrm{fm}^{-1}$ and $d=3 \mathrm{fm}$ was tried for the boson model with MT-III potential. Results for $\hat{S}^{\text {sym }}$ obtained using the TDTFE approach with this wave packet at time $t=64 \mathrm{fm}^{2}$ are shown in Fig. 9. For the fermion version, Fig. 10 presents the results for an initial wave packet with $q_{0}=1.0 \mathrm{fm}^{-1}$ and $d=2.5 \mathrm{fm}$. Results for the fermion version with this narrower momentum wave packet are of the same quality as the results obtained with the broader momentum wave packet. For the boson case, $\operatorname{Re} \hat{S}^{\text {sym }}$ and $\operatorname{Im} \hat{S}^{\text {sym }}$ are satisfactory for $0.4<q<1.75 \mathrm{fm}^{-1}$, although some oscillations showing up in the higher-energy region. There is some improvement in $\hat{S}^{\text {sym }}$ in the low-energy region. Wave-packet results for $\operatorname{Im} \hat{S}^{\text {sym }}$ now exhibit a dip at low-momentum values. However, it is still not as deep as the dip in the reference results. The inelasticity parameters and phase shifts implicit in Figs. 9 and 10 are shown in Figs. 11 and 12. It is gratifying to observe that there is considerable improvement in the low- $q$ behavior of the wave packet results for $\delta_{R}$. However, inelasticities for $q<0.5$ are still qualitatively wrong. Evidently, finite-element grids (in momentum space) used in these calculations are relatively coarse and unable to adequately represent time evolution of the wave-packets with small average momentum and narrow momentum distribution.

Numerical wave-packet solutions of the TDFE are considered next. Since the boson case provides a more stringent

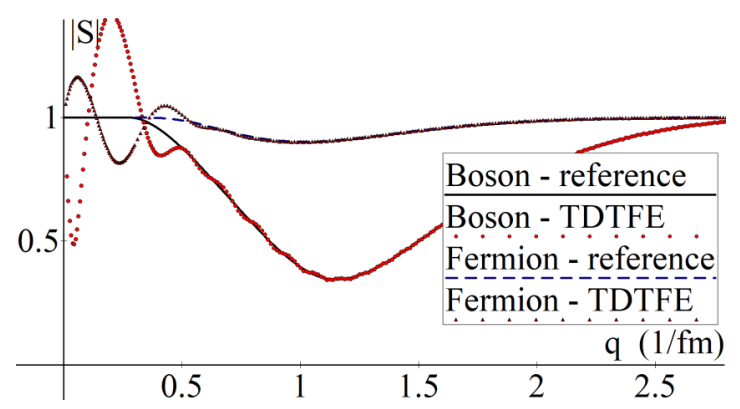

FIG. 8. Inelasticity parameter $|S|$ for three-boson and threefermion models with the MT-III potential. Parameters of the initial wave packet are $q_{0}=2 \mathrm{fm}^{-1}, y_{0}=9 \mathrm{fm}, d=1.5 \mathrm{fm}$. 


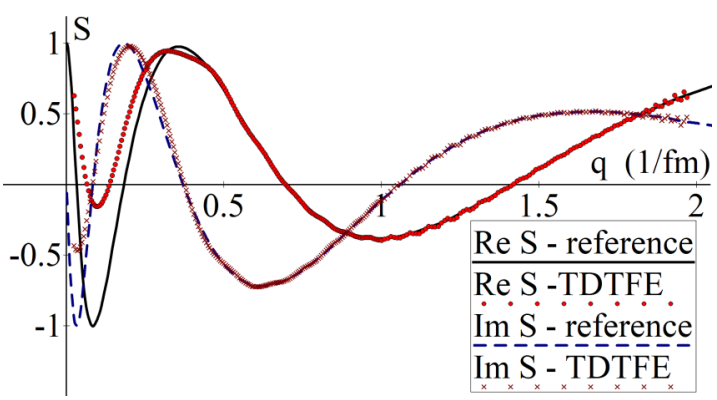

FIG. 9. Same as Fig. 5, but with $q_{0}=1 \mathrm{fm}^{-1}, y_{0}=18 \mathrm{fm}$, $d=3 \mathrm{fm}$.

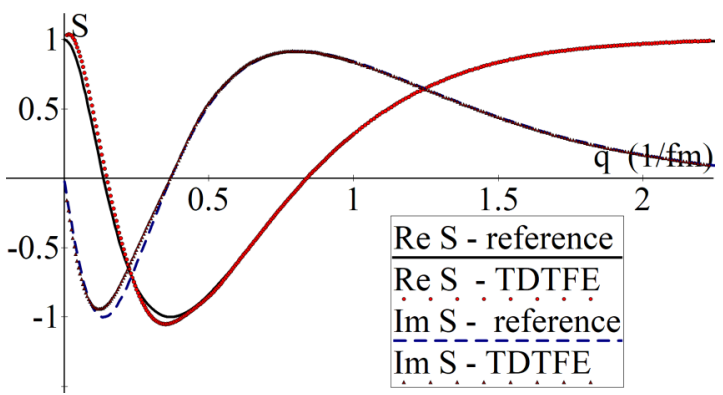

FIG. 10. Same as Fig. 6, but with $q_{0}=1 \mathrm{fm}^{-1}, y_{0}=9 \mathrm{fm}$, $d=2.5 \mathrm{fm}$.

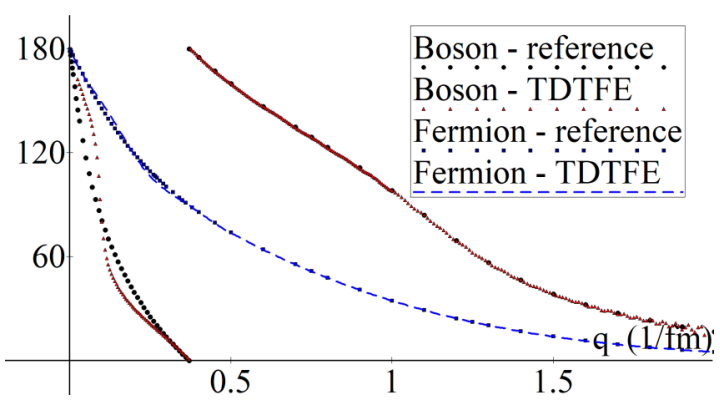

FIG. 11. Real part $\delta_{R}$ of phase shift for three-boson and threefermion models with the MT-III potential with the narrower momentum-space wave packets as in Figs. 9 and 10. This figure should be contrasted with Fig. 7.

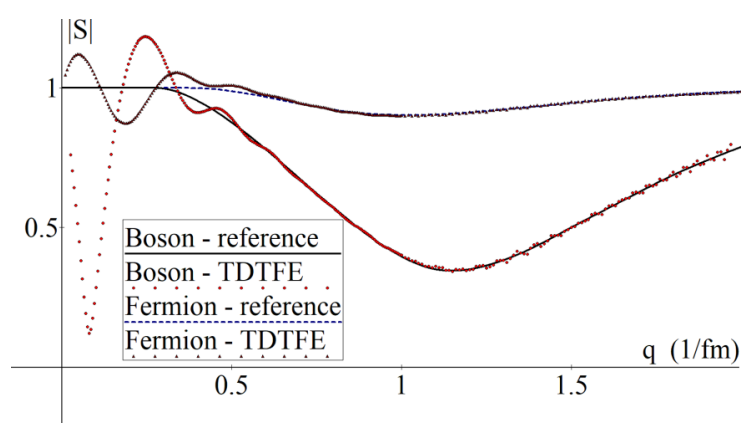

FIG. 12. Inelasticity parameter $|S|$ for three-boson and threefermion models with the MT-III potential with the narrower momentum-space wave packets as in Figs. 9 and 10. This figure should be contrasted with Fig. 8 .

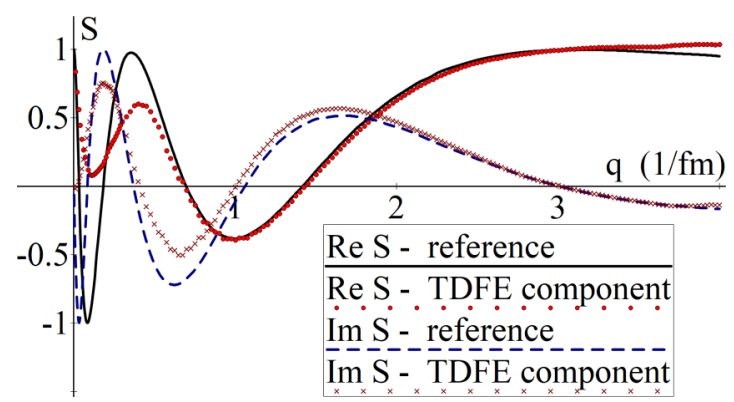

FIG. 13. Real and imaginary parts of $S^{\text {sym }}$ for the three-boson model with MT-III potential are plotted as a function of spectator momentum $q$. Wave-packet results are from calculations with timedependent Faddeev equations (TDFEs). $S$ matrices calculated via Eq. (55) from the Faddeev component of the TDFE are compared with the reference results. Initial wave-packet parameters are $q_{0}=$ $2 \mathrm{fm}^{-1}, y_{0}=9 \mathrm{fm}, d=1.5 \mathrm{fm}$.

testing system, calculations in the rest of this section are restricted to three-boson model with MT-III potential. $S$ matrices $\hat{S}^{\text {sym }}(E)$ extracted from numerically propagated Faddeev component at time $t=40$ are presented in Fig. 13. Since the same initial wave packet has been used in both TDFE and TDTFE calculations, Fig. 13 is to be compared with Fig. 5. Results from Faddeev component are not satisfactory for $q<1 \mathrm{fm}^{-1}$. Apparently spatial separation of the Faddeev components of the wave packet at asymptotic times that the formal analysis predicts does not get materialized in these calculations. But the sum of the Faddeev components provide more stable and more accurate results as shown in Fig. 14. In fact there are only slight differences between Figs. 5 and 14 for $S^{\mathrm{sym}}$. Nevertheless, based on this and similar other (unreported) calculations, it is concluded that the TDTFE approach appears to yield more accurate results than TDFE approach.

\section{SUMMARY AND OUTLOOK}

Present work reports the numerical wave-packet solution of the time-dependent Faddeev equations and successful calculation of rearrangement $S$ matrices for a wide range of energies from a single wave packet. To the best knowledge of this author, this constitutes the first instance where time-

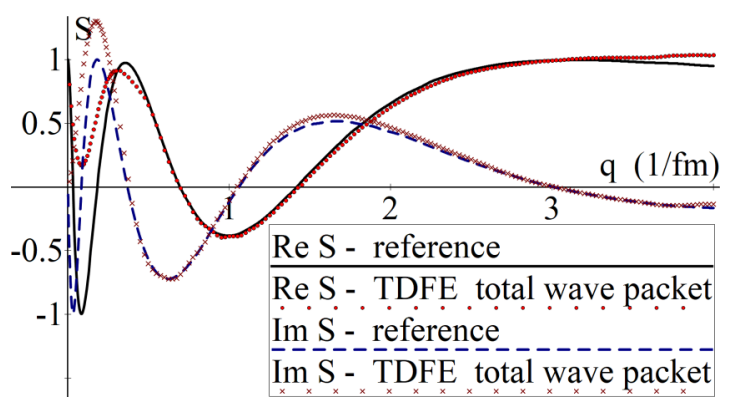

FIG. 14. Real and imaginary parts of $S^{\text {sym }}$ for the three-boson model are plotted as a function of spectator momentum $q$. Wavepacket results of this figure were obtained via Eq. (57) from the numerical total wave-packet solution of the TDFE (i.e., from the sum of Faddeev components). Same initial wave packet as in Fig. 13. 
dependent Faddeev equations are solved numerically. The local-potential model used to implement the proposed timedependent approach is a realistic one often used to test computational methods for three-body scattering [21-23,25]. The present work demonstrates that the time-dependent Faddeev approach is a promising addition to the the existing pool of computational methods for three-body scattering problems. Its computational advantage stems from the fact that one works entirely with square-integrable functions. The problems of boundary conditions or kernel singularities that besets the time independent approaches are avoided. That one can obtain scattering information for a range of collision energies from a single solution is perhaps the greatest advantage. In contrast, the computational effort needed for one collision energy in time-independent schemes is roughly the same magnitude as that needed by the propagation and analysis of just one wave packet. The computational effort involved in organizing and executing the propagation of one initial Faddeev wave packet is comparable to that of solving the Faddeev equations in coordinate space or AGS equations in momentum space for one collision energy. If anything, time-dependent approach is simpler as far as computational complexity is concerned because, once Hamiltonian matrix is constructed, the time propagation is reduced to repeated matrix vector multiplications and other basic linear algebra operations. As such, the main body of the calculation is in extremely suitable form for parallel computing with use of graphics processing units (GPU) [31,32]. By adopting the current method to computational platforms involving GPUs, more realistic potentials could be handled with finer discretization grids and without the drastic truncation of angular momentum states.

Compared with the time-dependent versions of CRC ansatz $[10,13]$ and hyperspherical-expansion approaches [6], the time-dependent Faddeev approach allows for the simultaneous use of Jacobi variables and bases for all rearrangements without the formal overcompleteness concern or the computational difficulties caused by the nonorthogonal overlap matrix.

Most Faddeev calculations in the time-independent context are staged in momentum space. Present study shows that the momentum space is rather convenient also in the timedependent context. As opposed to the moving and spreading character of wave packets in coordinate space, momentum space wave packets pretty much remain compact. Thanks to this feature, the numerical effort can be concentrated on the momentum domain where the packet has appreciable amplitude. One can use a finer grid over the effective support of the wave packet, while the rest of the computational domain can be covered with a coarser grid. This is also beneficial in connection with the boundary-reflection problem. It was observed that the use of a fine grid over a portion of the total computational domain has been sufficient to guarantee relatively long periods of reflection-free propagation. In fact, for the wave-packet calculations reported in Table II and Fig. 5, at $t=40 \mathrm{fm}^{2}$, the average distance of the spectator particle from the bound pair (as calculated from the numerical outgoing packet) comes out as $122.9 \mathrm{fm}$, while its dispersion is found as $29.5 \mathrm{fm}$. Note that the dispersion of $y$ in the initial wave packet was only $1.06 \mathrm{fm}$. Had one staged the calculation in the coordinate representation, the propagation of the wave packet until such long times would necessitate a very large computational domain in Jacobi coordinates and the use of absorbing potentials to combat the boundary reflection problem.

The present paper has concentrated on the extraction of rearrangement amplitudes from the wave-packet solutions of TDFE and TDTFE equations. Of course, these solutions also contain information about the breakup process. In fact, from the accuracy of rearrangement amplitudes one can infer that the total breakup probability at a given collision energy is predicted correctly. To tackle the problem of obtaining stateto-state breakup amplitudes, one observes that, at asymptotic future, the difference between the total wave packet and the sum of the rearrangement components can be identified as the outgoing packet for the breakup channel. That is, if one defines the breakup component of the total wave packet via $\left|\Psi_{\alpha n_{0} q_{0}}^{\text {(brekup) }}(t)\right\rangle=\left(I-\Pi_{1}-\Pi_{2}-\Pi_{3}\right)\left|\Psi_{\alpha n_{0} q_{0}}(t)\right\rangle$, then, for asymptotic times $T$, one should have $\left|\Psi_{\alpha n_{0} q_{0}}^{\text {(breap })}(T)\right\rangle=$ $S_{0 \alpha}\left|\Phi_{\alpha n_{0} q_{0}}(T)\right\rangle$, where $S_{0 \alpha}$ is the $S$ operator for the process $\alpha+(\beta \gamma) \rightarrow \alpha+\beta+\gamma$. Whether this provides a viable scheme for calculating state-to-state breakup amplitudes $\alpha<$ $p^{\prime} q^{\prime}\left|S_{0 \alpha}(E)\right| \phi_{n_{0}} q>_{\alpha}$ with $E=\epsilon+3 q^{2} / 4=p^{\prime 2}+3 q^{\prime 2} / 4$ will be the subject of future work.
[1] E. W. Schmid and H. Ziegelmann, The Quantum Mechanical Three-Body Problem (Pergamon Press, Oxford, 1974).

[2] W. Glockle, The Quantum Mechanical Few Body Problem (Springer, Berlin, 1983).

[3] O. A. Rubtsova, V. I. Kukulin, and V. N. Pomerantsev, Ann. Phys. (NY) 360, 613 (2015).

[4] J. Huang, S. Liu, D. H. Zhang, and R. V. Krems, Phys. Rev. Lett. 120, 143401 (2018).

[5] D. H. Zhang and H. Guo, Annu. Rev. Phys. Chem. 67, 135 (2016).

[6] J. Crawford and G. A. Parker, J. Chem Phys. 138, 054313 (2013).

[7] W. Hu and G. C. Schatz, J. Chem Phys. 125, 132301 (2006).

[8] J. Holz and W. Glockle, Phys. Rev. C 37, 1386 (1988).

[9] H. Kroger, R. J. Slobodrian, and G. L. Payne, Phys. Rev. C 37, 486 (1988).
[10] Z. C. Kuruoglu and F. S. Levin, Phys. Rev. Lett. 64, 1701 (1990).

[11] Z. C. Kuruoglu, Phys. Rev. A 42, 6314 (1990).

[12] Z. C. Kuruoglu and F. S. Levin, Phys. Rev. A 46, 2304 (1992).

[13] Z. C. Kuruoglu, in Time Dependent Quantum Molecular Dynamics, edited by J. Broeckhove (Plenum Press, NATO ASI Series, 1992). pp. 167-179.

[14] Z. C. Kuruoglu and F. S. Levin, Phys. Rev. C 36, 49 (1987).

[15] G. L. Payne, W. Glockle, and J. L. Friar, Phys. Rev. C 61, 024005 (2000).

[16] D. J. Kouri, H. Kruger, and F. S. Levin, Phys. Rev. D 15, 1156 (1977).

[17] J. W. Evans, J. Math. Phys. 22, 1672 (1981).

[18] J. W. Evans, D. K. Hoffman, and D. J. Kouri, J. Math. Phys. 24, 576 (1981). 
[19] R. A. Sultanov, W. Sandhas, and V. B. Belyaev, Eur. Phys. J. D 5, 33 (1999).

[20] R. A. Malfliet and J. A. Tjon, Nucl. Phys. 127, 161 (1969).

[21] J. L. Friar et al., Phys. Rev. C 42, 1838 (1990).

[22] J. L. Friar, G. L. Payne, W. Glockle, D. Huber, and H. Witala, Phys. Rev. C 51, 2356 (1995).

[23] E. Garrido, A. Kievsky, and M. Viviani, Phys. Rev. C 90, 014607 (2014).

[24] Z. C. Kuruoglu, Phys. Rev. A 44, 7307 (1991).

[25] Z. C. Kuruoglu, Phys. Rev. C 44, 1354 (1991).
[26] L. D. Faddeev, Zh. Eksp. Teor. Fiz. 39, 1459 (1960) [Sov. Phys. JETP 12, 1014 (1961)].

[27] F. S. Levin, Phys. Rev. C 21, 2199 (1980).

[28] N. M. Larson and J. H. Hetherington, Phys. Rev. C 9, 699 (1974).

[29] W. M. Kloet and J. A. Tjon, Ann. Phys. (NY) 79, 407 (1973).

[30] P. M. Prenter, Splines and Variational Methods (Wiley, New York, 1975).

[31] V. N. Pomerantsev, V. I. Kukulin, O. A. Rubtsova, and S. K. Sakhiev, Comput. Phys. Comm. 204, 121 (2016).

[32] P.-Y. Zhang and K.-L. Han, J. Phys. Chem. A 117, 8512 (2013). 